Daniel Aubram, Stavros A. Savidis, Frank Rackwitz

\title{
Vibro-Injection Pile Installation in Sand: Part I-Interpretation as Multi-material Flow
}

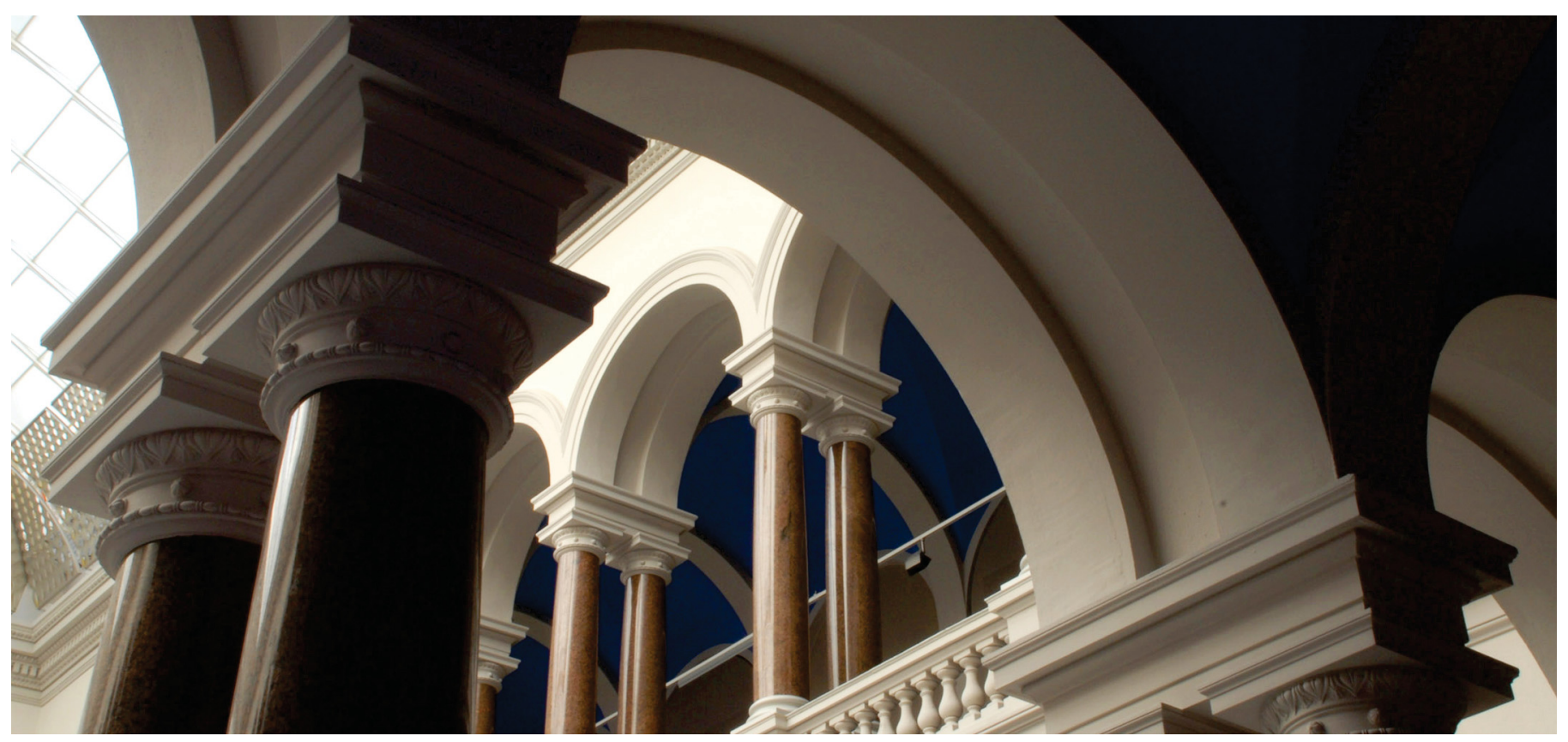

Aubram, D., Rackwitz, F., \& Savidis, S. A. (2015). Vibro-Injection Pile Installation in Sand: Part IInterpretation as Multi-material Flow. In: Triantafyllidis T. (eds) Holistic Simulation of Geotechnical Installation Processes. Lecture Notes in Applied and Computational Mechanics, vol 77. Springer, Cham. pp. 73-102. https://doi.org/10.1007/978-3-319-18170-7_5 


\title{
Vibro-Injection Pile Installation in Sand: 1. Interpretation as Multi-Material Flow
}

\author{
Daniel Aubram ${ }^{1}$, Frank Rackwitz ${ }^{2}$, and Stavros A. Savidis ${ }^{1}$ \\ 1 Chair of Soil Mechanics and Geotechnical Engineering, Technische Universität \\ Berlin, Secr. TIB1-B7, Gustav-Meyer-Allee 25, D-13355 Berlin, Germany \\ 2 Department of Geotechnical Engineering, Ostbayerische Technische Hochschule \\ Regensburg, Prüfeninger Straße 58, D-93049 Regensburg, Germany
}

\begin{abstract}
The installation of vibro-injection piles into saturated sand has a significant impact on the surrounding soil and neighboring buildings. It is generally characterized by a multi-material flow with large material deformations, non-stationary and new material interfaces, and by the interaction of the grain skeleton and the pore water. Part 1 in this series of papers is concerned with the mathematical and physical modeling of the multi-material flow associated with vibro-injection pile installation. This model is the backbone of a new multi-material arbitrary Lagrangian-Eulerian (MMALE) numerical method presented in Part 2.
\end{abstract}

Keywords: multi-material flow, large deformations, mixture, soil mechanics, sand, averaging, homogenization, closure law, arbitrary Lagrangian-Eulerian

\section{Introduction}

Vibro-injection piles, in German called "Rüttelinjektionspfähle (RI-Pfähle)", are used in sandy soil to tie back the base slab of deep excavations in urban area with high groundwater level. They consist of an H-section steel pile equipped with an injection tube and a welded-on collar located at the pile toe (Figs. 1 and 2). During the installation of the pile into the water-saturated sand by vibration the soil loses its shear strength ("soil liquefaction") and the annular gap generated by the collar is continuously injected with grout. The installation process of a vibro-injection pile interacts to a great extent with its neighborhood $[32,34]$. This is why the numerical modeling of the installation process is of high practical relevance for the realistic prediction of the deformations and the load bearing behavior of the wall.

Sufficiently realistic computational models must be able to reproduce the basic installation phenomena (Fig. 2, detail A). These include the shearing and liquefaction of the locally undrained saturated soil, the displacement of the liquefied soil by the pile and the grout, as well as the mixing of the grout with the liquefied soil. Stated more generally, the installation of vibro-injection piles can be characterized by a multi-material flow with large material deformations, by free surfaces and non-stationary contact interfaces, and by the complex coupled

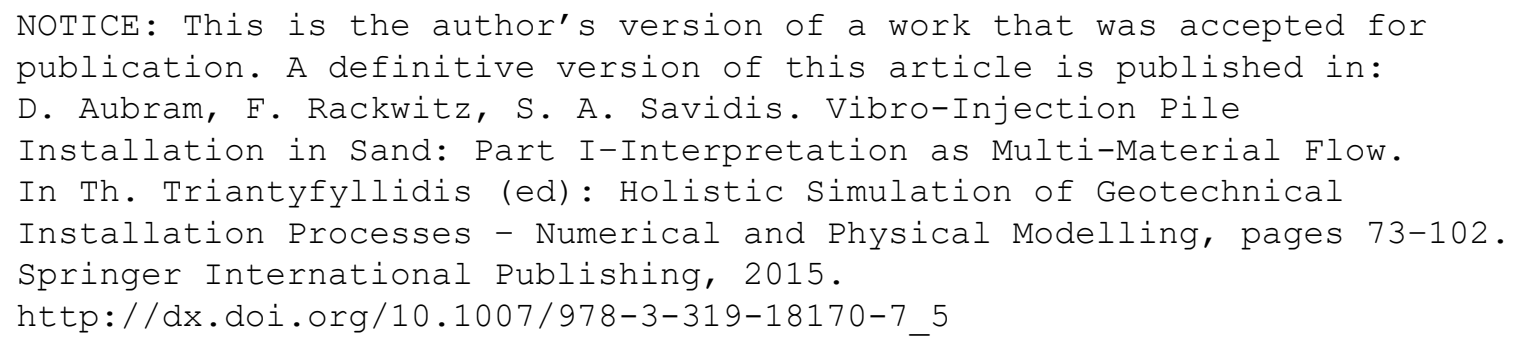



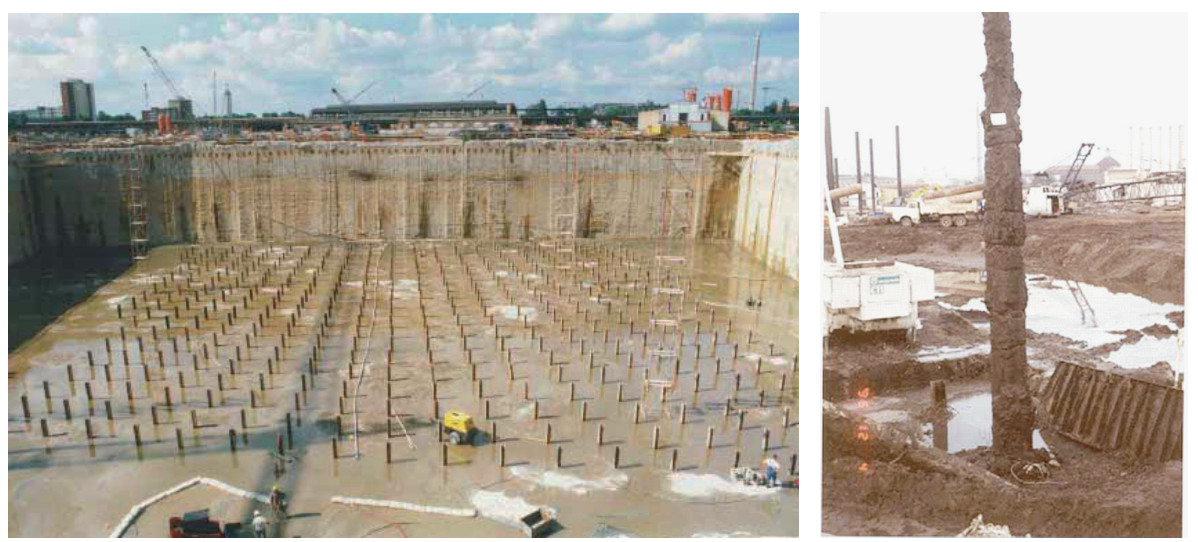

Fig. 1. Grid of vibro-injection piles to tie back an excavation base slab. Deep excavation of about $20 \mathrm{~m}$ in urban area (left) and recovered pile (right).

behavior of the grain skeleton and the pore water. The numerical simulation of such problems is very challenging. Even the penetration of the steel profile alone could not be handled by using the classical Lagrangian formulations of the finite element method (FEM) [47, 44]. It is no surprise, therefore, that up to date there are no FE prediction models for the installation of RI-piles and for the related time-histories of the stress and density states within the soil.

It is the aim of the Subproject 5 as part of the DFG Research Unit FOR 1136 to make a significant contribution to this area through the development of a so-called multi-material arbitrary Lagrangian-Eulerian (MMALE) method. Objectives are, firstly, to predict both qualitatively and quantitatively the stress and density time-histories within the soil in the vicinity of the vibro-injection pile and hence the effects of its installation process on close-by structures and, secondly, the realistic simulation of the load bearing and deformation behavior of the completed pile. Based on a continuum mechanical description, especially the single installation phenomena (driving of the steel profile by vibration, liquefaction and displacement of the undrained soil during vibratory pile driving, grouting of the emerging annular gap between the steel profile and the liquefied soil; see also Fig. 2) should be modeled by using an MMALE finite element method.

MMALE methods fall into the category of arbitrary Lagrangian-Eulerian methods $[23,35,4,2]$ and have no limitations concerning material deformations and the evolution or generation of material interfaces. The mesh can move independently of the material such that material interfaces (boundaries) may flow through the mesh. Elements cut by interfaces contain a mixture of two or more materials and are referred to as multi-material elements. The mixture is treated as an effective single-phase material or homogenized mixture on the element level. Hence, quantities related to each material must be "mixed" in a certain way to yield the corresponding homogenized element quantities. As the latter 

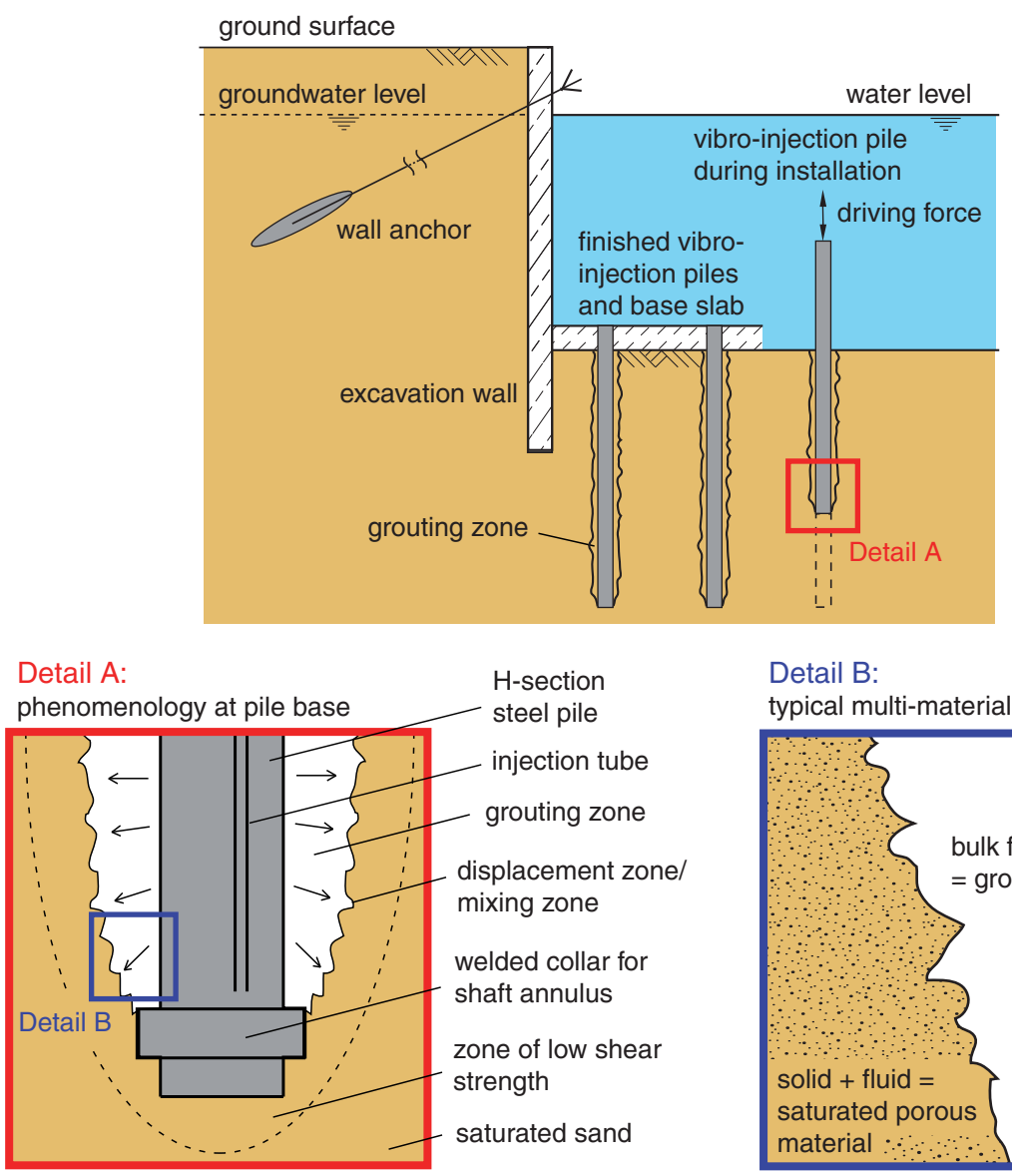

Detail B:

typical multi-material zone

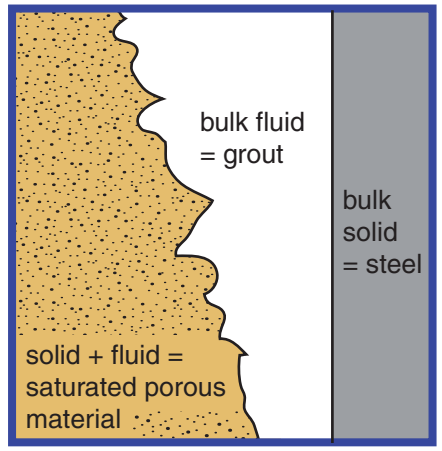

Fig. 2. Illustration of the installation of a vibro-injection pile in saturated sand to tie back an excavation base slab. Detail A: phenomenology at the pile base, Detail B: typical zone of the considered multi-material flow including macroscopic interfaces. 
should be based on physical principles, the development of an MMALE method for vibro-injection pile installation in sand has to start with the mathematical and physical modeling of the associated multi-material flow, which is the content of Part 1 in this series of papers. Details of the new method and of our numerical and experimental investigations concerning vibro-injection pile installation will be presented in Part 2.

Part 1 is structured as follows. In Section 2 we introduce the three different spatial scales (microscale, mesoscale, macroscale) of the continuum mechanical problem and formulate the balance principles and jump conditions of the microscopic multi-material flow. This flow is homogenized in Section 3 to yield a macroscopic multi-material flow forming the physical model of the numerical method. One of the basic techniques to achieve this homogenization is spatial averaging, a theory which is widely used in the field of multiphase flow and the modeling of porous media. Section 4 then addresses the closure of the resulting system of equations by making reasonable assumptions and establishing interfacial transfer laws, constitutive laws, and topological laws. The final form of the macroscopic model for multi-material flow is derived in Section 5 through model reduction based on additional, a priori closure assumptions. The paper closes with concluding remarks and outlook in Section 6 .

\section{Spatial Scales and Balance Principles}

\subsection{Three Spatial Scales}

Consider the process of vibro-injection pile installation into sand as illustrated in Fig. 2. Detail B of that figure can be regarded as a still image of a multimaterial flow recorded through a spatially fixed observation window. We take this zone as characteristic of the multi-material (multi-constituent) flow and assume that the basic features of the flow are independent of the specific arrangement resp. distribution of the constituents. The characteristic zone is filled with an immiscible mixture consisting of a bulk solid phase (steel), a bulk fluid phase (grout), and a compound phase consisting of a solid species and a fluid species which represents the fluid-saturated porous material (sand) - the term "species" is used here to distinguish these constituents from the bulk solid and bulk fluid phases. Void (empty space) is considered as a particular bulk fluid phase in our model. Immiscibility of the mixture is characterized by the fact that the constituents are separated by interfaces.

Three spatial scales are introduced in accordance with [6]; see Fig. 3. The porous material is constituted by an assembly of sand grains, whose typical diameter defines the microscale of the problem, $l_{\text {micro }}$. However, in the present research we have to properly reproduce the nonlinear coupled mechanical behavior of the water-saturated sand. In this regard the best models currently available are phenomenological two-phase models that rely on a continuum representation of the soil and not on micromechanics. Therefore, we introduce the characteristic length at which the saturated sand can be represented by a continuum as the mesoscale $l_{\text {meso }}$. A similar issue of upscaling, but on a larger scale, 

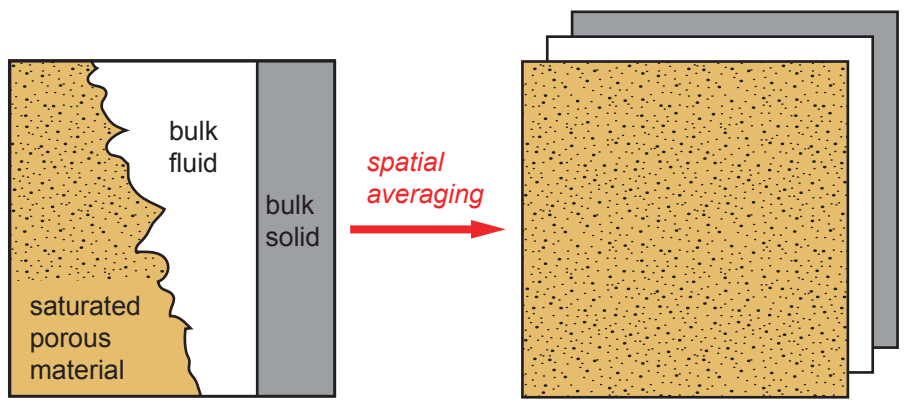

representative volume element of mixture

homogeneous mixture of bulk solid, bulk fluid, and saturated porous material

Macroscale

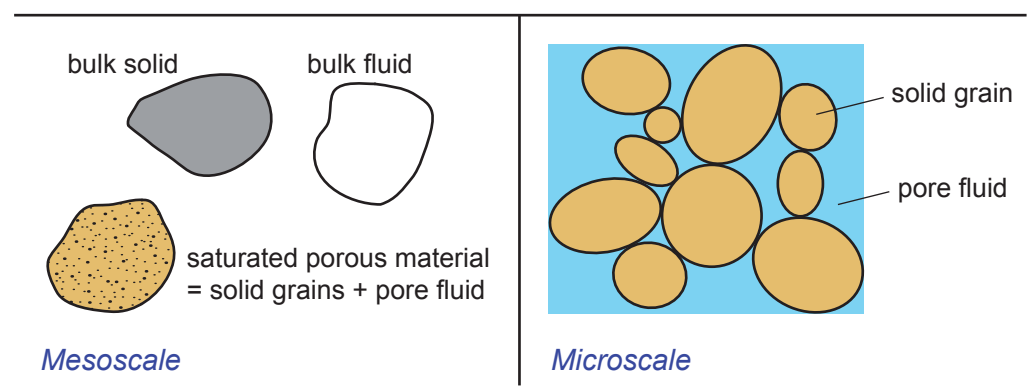

Fig. 3. Three spatial scales to model vibro-injection pile installation in saturated sand. 
has to be faced in multi-material elements of the MMALE method. Accordingly, we postulate the existence of a representative volume element (RVE) at some macroscale, $l_{\text {macro }}$, through which the immiscible mixture of mesoscale continua (bulk solid, bulk fluid, and saturated sand) can be equivalently modeled as an effective single-phase material (homogenized immiscible mixture) treated by the MMALE method. The real world is modeled on an even larger scale; in the literature this is sometimes called the megascale.

To make this point clear, we remark that our definitions of a constituent, a phase, and a species is comparable to those generally used in chemistry and thermodynamics. Constituents are called the individual (chemically-independent) materials composing the mixture on the microscale (constituent $=$ micro-continuum). The heterogeneous mixture consists of different material phases separated by macroscopic interfaces. A phase is physically distinct and mechanically separable and, in our case, might be identified with a constituent if there would be no saturated porous medium in the mixture. However, we generally define a phase to be a compound material consisting of solid species and a fluid species, and which can be regarded homogeneous on a mesoscale (phase = meso-continuum). Finally, as indicated by Fig. 3, on the postulated macroscale the mixture of phases can be addressed as a homogeneous mixture (macrocontinuum).

\subsection{Microscopic Balance Principles}

On the microscale all constituents of the mixture can be regarded as microcontinua (Fig. 3), governed by the equations of continuum mechanics [38, 39, $27,28,20,1]$. Generally these include conservation of mass

$$
\frac{\partial \rho}{\partial t}+\operatorname{div}(\rho \boldsymbol{v})=0
$$

and balance of momentum

$$
\frac{\partial \rho \boldsymbol{v}}{\partial t}+\operatorname{div}(\rho \boldsymbol{v} \otimes \boldsymbol{v})=\rho \boldsymbol{b}+\operatorname{div} \boldsymbol{\sigma}
$$

assumed to hold in a domain of interest $\mathcal{V} \subset \mathcal{S}$ in the ambient Euclidian space and time interval $[0, T] \subset \mathbb{R}$. In the equations, $\boldsymbol{v}$ denotes the material velocity in the spatial description, $\rho$ is the mass density, $\boldsymbol{b}$ is a prescribed body force per unit mass (e.g. gravity), $\boldsymbol{\sigma}=\boldsymbol{\sigma}^{\mathrm{T}}$ is the symmetric Cauchy stress, div is the spatial divergence operator, and $\otimes$ is the tensor product. The notation is found at the end of this paper.

In the process of vibro-injection pile installation, the velocities are moderate and thermal effects can be ignored, so that equations (1) and (2) alone serve as the balance principles of that initial boundary value problem. At significantly larger velocities, on the other hand, thermal and strain rate effects may constitute an important part of the solution. In this case balance of energy must be added to the set of governing equations. 
While the balance principles hold in the interior of each constituent, the jump conditions for mass and momentum given by

$$
\llbracket \rho\left(\boldsymbol{v}-\boldsymbol{v}_{\mathrm{I}}\right) \rrbracket \cdot \boldsymbol{n}^{*}=0 \quad \text { and } \quad \llbracket \rho \boldsymbol{v} \otimes\left(\boldsymbol{v}-\boldsymbol{v}_{\mathrm{I}}\right)-\boldsymbol{\sigma} \rrbracket \cdot \boldsymbol{n}^{*}=\mathbf{0},
$$

respectively, must hold at the interfaces; the jump conditions express the balance principles at an interface. Here - denotes the single contraction of tensors and $\llbracket q \rrbracket \stackrel{\text { def }}{=} q^{[l]}-q^{[k]}$ the jump of $q$ across the interface between material $l$ and material $k$, with $q^{[k]}$ being the limit value of $q$ on the $k$-side of the interface, $\boldsymbol{v}_{\mathrm{I}}$ is the interface velocity, and $\boldsymbol{n}^{*}$ is the field of normals on the interface pointing outward of the $k$-th micro-continuum. In the jump condition for momentum, $(3)_{2}$, no surface tension is taken into account.

We remark that the balance principles (1) and (2) are in the so-called Eulerian conservation form, and that all equations in this section refer to a spatial reference volume instantaneously occupied by the material on the microscale. The quantities $\rho(x, t), \boldsymbol{v}(x, t)$, etc., represent microscopic (non-averaged) timedependent spatial fields, with $x \in \mathcal{V}$ and $t \in[0, T]$. This means that a spatial point is viewed as being currently occupied by a single constituent.

\section{Macroscopic Multi-Material Flow}

\subsection{Fundamentals of Spatial Averaging}

In our approach the effective multi-material dynamics on the macroscale is derived from physical principles by making use of spatial averaging $[42,20,26,6]$. Spatial averaging results in continuum mechanical equations which are similar to those that can be derived using the continuum theory of mixtures and the theory of porous media [38, 16, 22, 18]; see also Subproject 2 of this research unit. However, the advantage of the averaging approach is that information available at a smaller scale is transferred to the larger scale with respect to which averaging is carried out.

Let $\mathcal{V}^{\prime} \subset \mathcal{V}$ be a time-independent RVE having the characteristic length $l_{\text {macro }}$, and $q(x, t)$ be an arbitrary time-dependent spatial microscopic field for all $x \in \mathcal{V}$ and $t \in[0, T]$. Then, the volume average of $q$ is defined through

$$
\langle q\rangle(x, t) \stackrel{\text { def }}{=} \frac{1}{V^{\prime}} \int_{\mathcal{V}^{\prime}} q(x+\boldsymbol{\xi}, t) \mathrm{d} v .
$$

Here $\mathrm{d} v$ is the volume form on $\mathcal{S}$ and $V^{\prime} \stackrel{\text { def }}{=} \int_{\mathcal{V}^{\prime}} \mathrm{d} v$ is the volume measure of $\mathcal{V}^{\prime}$. Moreover, $\boldsymbol{\xi}=x^{\prime}-x$ and $x^{\prime} \in \mathcal{V}^{\prime}$. By (4) and the definition of the RVE, averaged quantities always refer to the macroscale.

A phase function (or indicator function) is defined by

$$
P^{k}(x, t) \stackrel{\text { def }}{=} \begin{cases}1 & \text { if } x \text { is in phase } k \text { at time } t \\ 0 & \text { else }\end{cases}
$$


for all $x \in \mathcal{V}$ and $t \in[0, T]$, where $k \in\left\{1, \ldots, n_{\text {mat }}\right\}$ and $n_{\text {mat }}$ being the number of phases in the mixture. The phase function is a so-called generalized function resp. distribution and picks out the generally time-dependent $k$-phase volume $\mathcal{V}^{k} \subset \mathcal{V}^{\prime}$. By the definition of a volume average (4), then, the volume fraction of the $k$-th phase with respect to $\mathcal{V}^{\prime}$ is defined through

$$
f^{k} \stackrel{\text { def }}{=}\left\langle P^{k}\right\rangle=\frac{1}{V^{\prime}} \int_{\mathcal{V}^{\prime}} P^{k} \mathrm{~d} v=\frac{1}{V^{\prime}} \int_{\mathcal{V}^{k}} \mathrm{~d} v=\frac{V^{k}}{V^{\prime}} \quad \in[0,1]
$$

where $V^{k} \stackrel{\text { def }}{=} \int_{\mathcal{V}^{k}} \mathrm{~d} v$. Equation (6) is a natural, that is, derived definition of volume fraction. By contrast, volume fraction is postulated in the continuum theory of mixtures and the theory of porous media.

We now assume that all material phases of the considered mixture are composed of a solid species (denoted by $\beta=\mathrm{s}$ ) and a fluid species (denoted by $\beta=\mathrm{f}$ ). In fact the portion of one of these species in a particular phase might be zero. For all $x \in \mathcal{V}$ and $t \in[0, T]$, we then define another indicator function $S^{\beta}$, called species function, which equals zero everywhere except on the spatial region occupied by the $\beta$-species at time $t$, where it is equal to one. Concerning the RVE, we denote this region by $\mathcal{V}^{\beta} \subset \mathcal{V}^{\prime}$. Therefore, by the property of indicator functions, the product $P^{k} S^{\beta}$ is the phase-species function picking out the partial volume occupied by the $\beta$-species in the $k$-phase:

$$
\left(P^{k} S^{\beta}\right)(x, t)= \begin{cases}1 & \text { if } x \in \mathcal{V} \text { is in species } \beta \text { of phase } k \text { at } t \in[0, T] \\ 0 & \text { else. }\end{cases}
$$

The volume fraction of the $\beta$-species in the $k$-phase is then obtained from

$$
\pi^{\beta k} \stackrel{\text { def }}{=} \frac{\left\langle P^{k} S^{\beta}\right\rangle}{f^{k}}=\frac{1}{f^{k} V^{\prime}} \int_{\mathcal{V}^{\prime}} P^{k} S^{\beta} \mathrm{d} v=\frac{1}{V^{k}} \int_{\mathcal{V}^{\beta k}} \mathrm{~d} v=\frac{V^{\beta k}}{V^{k}} \quad \in[0,1],
$$

where $\mathcal{V}^{\beta k} \stackrel{\text { def }}{=} \mathcal{V}^{k} \cap \mathcal{V}^{\beta}$ and $V^{\beta k} \stackrel{\text { def }}{=} \int_{\mathcal{V}^{\beta k}} \mathrm{~d} v$ is the volume of the $\beta$-species in the $k$-phase in the RVE. The macroscopic $\beta$-species fraction in the mixture is $\pi^{\beta} \stackrel{\text { def }}{=} \sum_{k} f^{k} \pi^{\beta k}$, and

$$
\sum_{k} f^{k}=1 \quad \text { and } \quad \sum_{\beta} \pi^{\beta k}=1 .
$$

As we are concerned with phases solely composed of a solid species and a fluid species, we simply define the fluid fraction (or porosity) of the $k$-phase through

$$
n^{k} \stackrel{\text { def }}{=} \pi^{\mathrm{f} k}
$$

so that the solid fraction within the $k$-phase becomes $\pi^{\mathrm{s} k}=1-n^{k}$ by using $(9)_{2}$. If the $k$-phase consists of a solid without significant porosity (e.g. steel), then $n^{k}=0$. If on the other hand the $k$-phase is a fluid, then $n^{k}=1$ applies. Pure solid mixtures are characterized by $n^{k}=0$ and pure fluid mixtures by 
$n^{k}=1$ for all $k \in\left\{1, \ldots, n_{\text {mat }}\right\}$, that is, $n \stackrel{\text { def }}{=} \sum_{k} n^{k}=0$ or 1 respectively. The mixture represented by a single fluid-saturated porous medium is characterized by $f^{k}=1\left(n_{\text {mat }}=1\right)$ and $0<n^{k}<1$.

Spatial averaging of microscopic physical fields on the constituents is done in a similar way as in (8) in conjunction with (4) and (6). For example, the averaged mass density and velocity of the solid species in the $k$-phase are given by

$$
\rho^{\mathrm{s} k} \stackrel{\text { def }}{=} \frac{\left\langle P^{k} S^{\mathrm{s}} \rho\right\rangle}{f^{k}\left(1-n^{k}\right)} \quad \text { and } \quad \boldsymbol{v}^{\mathrm{s} k} \stackrel{\text { def }}{=} \frac{\left\langle P^{k} S^{\mathrm{s}} \rho \boldsymbol{v}\right\rangle}{f^{k}\left(1-n^{k}\right) \rho^{\mathrm{sk}}},
$$

indicating that $\boldsymbol{v}^{\text {sk }}$ in fact is a mass-weighted volume average. While $\rho^{\text {sk }}$ is the intrinsic (or material) mass density, the bulk mass density of the $k$-phase solid species with respect to the RVE is obtained from $f^{k}\left(1-n^{k}\right) \rho^{\mathrm{s} k}$. The mass density of the mixture, by the properties (9), can be computed from

$$
\langle\rho\rangle=\sum_{k} \sum_{\beta} f^{k} \pi^{\beta k} \rho^{\beta k}=\sum_{k} f^{k} \rho^{k}=\sum_{k} f^{k}\left(\left(1-n^{k}\right) \rho^{\mathrm{s} k}+n^{k} \rho^{\mathrm{f} k}\right),
$$

where $\rho^{k}$ is the (intrinsic) mass density of the $k$-phase in the mixture. As an example, consider a specimen of dry sand in which the pores are filled with gas of negligible density such that $\rho^{\mathrm{f} k} \approx 0$ and $n_{\text {mat }}=1$. Then, $\rho^{\mathrm{s} k}=\rho^{\mathrm{s}}$ represents the grain mass density and $(1-n) \rho^{\mathrm{s}} \approx\langle\rho\rangle$ is the bulk mass density, which is approximately equal to the mass density $\langle\rho\rangle$ of the solid-gas mixture.

We emphasize that $k, \beta$, etc., are labels and not coordinate indices. No summation on repeated labels in a term is enforced unless the sigma notation is employed.

Based on (11) and (12) the mean spatial velocity of the mixture is related to the momentum and mass densities of the constituents by

$$
\langle\boldsymbol{v}\rangle=\frac{\sum_{k} \sum_{\beta} f^{k} \pi^{\beta k} \rho^{\beta k} \boldsymbol{v}^{\beta k}}{\sum_{k} \sum_{\beta} f^{k} \pi^{\beta k} \rho^{\beta k}}=\frac{\sum_{k} f^{k} \rho^{k} \boldsymbol{v}^{k}}{\sum_{k} f^{k} \rho^{k}}=\langle\rho\rangle^{-1} \sum_{k} f^{k} \rho^{k} \boldsymbol{v}^{k},
$$

where

$$
\boldsymbol{v}^{k} \stackrel{\text { def }}{=} \frac{\left\langle P^{k} \rho \boldsymbol{v}\right\rangle}{f^{k} \rho^{k}}=\sum_{\beta} \frac{f^{k} \pi^{\beta k} \rho^{\beta k}}{f^{k} \rho^{k}} \boldsymbol{v}^{\beta k}=\frac{1}{\rho^{k}}\left(\left(1-n^{k}\right) \rho^{\mathrm{sk}} \boldsymbol{v}^{\mathrm{sk}}+n^{k} \rho^{\mathrm{fk}} \boldsymbol{v}^{\mathrm{f} k}\right)
$$

is the mean spatial velocity of the $k$-phase. It should be pointed out here that averaged quantities are defined for all $x \in \mathcal{V}$ and $t \in[0, T]$. In other words, the mixture after averaging is represented by a superposition of continuous bodies with independent motions (i.e. overlapping and interpenetrating continua), as in the continuum theory of mixtures according to [38].

Averaging of the stress field is not straightforward; see [6], or [26, ch. 2] for the case where $S^{\beta} \equiv 1$. For example, the averaged microscopic Cauchy stress within the solid species of the $k$-phase is given by

$$
\boldsymbol{\sigma}^{\mathrm{s} k} \stackrel{\text { def }}{=} \frac{\left\langle P^{k} S^{\mathrm{s}} \boldsymbol{\sigma}\right\rangle}{f^{k}\left(1-n^{k}\right)}-\frac{\left\langle P^{k} S^{\mathrm{s}} \rho \overline{\boldsymbol{v}}^{\mathrm{s} k} \otimes \overline{\boldsymbol{v}}^{\mathrm{s} k}\right\rangle}{f^{k}\left(1-n^{k}\right)},
$$


in which $\overline{\boldsymbol{v}}^{\mathrm{s} k}$ denotes the deviation of the microscopic velocity of the solid species in the $k$-phase from its spatial average $\boldsymbol{v}^{\mathrm{s} k}$. If, as in the case considered here, the solid species of the $k$-phase is formed by a grain skeleton, then $\boldsymbol{\sigma}^{\text {sk }}$ represents the microscopic Cauchy stress within the grains averaged with respect to the RVE. The second term on the right hand side of (15) is a residual stress, called Reynolds stress, due to mass flux relative to the averaging volume. The averaged stress in the grain skeleton, $\boldsymbol{\sigma}^{\mathrm{s} k}$, should not be confused with Terzaghi's effective stress whose proper definition is given below.

Our three-scale approach to obtain the macroscopic fields differs significantly from that in [6]. Consider a representative volume element $\mathcal{V}^{\prime \prime}$ smaller than $\mathcal{V}^{\prime}$ for each phase $k$ having the characteristic length $l_{\text {meso }}$ at which the mixture of the two species $\beta \in\{\mathrm{s}, \mathrm{f}\}$ in the $k$-phase can be represented by a continuum. Then, spatial averaging over $\mathcal{V}^{\prime}$ after averaging over $\mathcal{V}^{\prime \prime}$ will result in the same averaged (i.e. macroscopic) physical field as spatial averaging over $\mathcal{V}^{\prime}$ alone. Therefore, we do not introduce a separate operator for averaging over $\mathcal{V}^{\prime \prime}$, as done in [6], so that stressing the term "mesoscale" is somewhat superfluous; the averaged equations of a binary mixture are obtained from those presented herein simply by setting $P^{k} \equiv 1$ (resp. $f^{k} \equiv 1$ ). In our approach we use only one macroscopic averaging operator in conjunction with a composition of indicator functions, called the phase-species function, which picks out a particular species in a particular phase. A detailed comparison our approach and that in [6] is left to future work.

\subsection{Macroscopic Balance Principles for Each Species}

By using spatially averaged fields and taking into account basic averaging theorems, the macroscopic (averaged) balance principles for each species in the mixture can be derived. In particular, conservation of mass and balance of momentum of the $\beta$-th species of the $k$-th phase with respect to the whole mixture in the representative volume element read

$$
\begin{aligned}
\frac{\partial f^{k} \pi^{\beta k} \rho^{\beta k}}{\partial t}+\operatorname{div}\left(f^{k} \pi^{\beta k} \rho^{\beta k} \boldsymbol{v}^{\beta k}\right)= & \Pi^{\beta k} \\
\frac{\partial f^{k} \pi^{\beta k} \rho^{\beta k} \boldsymbol{v}^{\beta k}}{\partial t}+\operatorname{div}\left(f^{k} \pi^{\beta k} \rho^{\beta k} \boldsymbol{v}^{\beta k} \otimes \boldsymbol{v}^{\beta k}\right)= & f^{k} \pi^{\beta k} \rho^{\beta k} \boldsymbol{b}^{\beta k}+\operatorname{div}\left(f^{k} \pi^{\beta k} \boldsymbol{\sigma}^{\beta k}\right) \\
& +\Pi^{\beta k} \overline{\boldsymbol{v}}_{\mathrm{I}}^{\beta k}+\boldsymbol{\Gamma}^{\beta k}
\end{aligned}
$$

in which $\beta \in\{\mathrm{s}, \mathrm{f}\}, k \in\left\{1, \ldots, n_{\text {mat }}\right\}$, and

$$
\begin{gathered}
\Pi^{\beta k} \stackrel{\text { def }}{=}\left\langle\delta_{\mathrm{I}}^{\beta k} \rho^{[\beta k]}\left(\boldsymbol{v}-\boldsymbol{v}_{\mathrm{I}}\right)^{[\beta k]} \cdot \boldsymbol{n}^{\beta k *}\right\rangle, \\
\Pi^{\beta k} \overline{\boldsymbol{v}}_{\mathrm{I}}^{\beta k} \stackrel{\text { def }}{=}\left\langle\delta_{\mathrm{I}}^{\beta k} \rho^{[\beta k]} \boldsymbol{v}^{[\beta k]} \otimes\left(\boldsymbol{v}-\boldsymbol{v}_{\mathrm{I}}\right)^{[\beta k]} \cdot \boldsymbol{n}^{\beta k *}\right\rangle, \\
\text { and } \quad \boldsymbol{\Gamma}^{\beta k} \stackrel{\text { def }}{=}-\left\langle\delta_{\mathrm{I}}^{\beta k} \boldsymbol{\sigma}^{[\beta k]} \cdot \boldsymbol{n}^{\beta k *}\right\rangle .
\end{gathered}
$$

$\langle\cdot\rangle$ is the spatial average as defined by $(4), \delta_{\mathrm{I}}^{\beta k}$ is a Dirac delta function which picks out the interface of the $\beta$-th species of the $k$-th phase, and $\boldsymbol{n}^{\beta k *}$ is the field 
of outward normals on that interface. Moreover, for the microscopic field $q, q^{[\beta k]}$ is the restriction to the side of the interface lying in the $\beta$-species of the $k$-phase.

The terms $\Pi^{\beta k}, \Pi^{\beta k} \overline{\boldsymbol{v}}_{\mathrm{I}}^{\beta k}$, and $\boldsymbol{\Gamma}^{\beta k}$ are due to the interaction between the species and phases and can be interpreted as follows. $\Pi^{\beta k}$ describes the (average) mass transfer onto the $\beta$-species of the $k$-phase by all other constituents of the mixture through chemical reactions or erosion, for example. The momentum transfer term $\boldsymbol{\Gamma}^{\beta k}$ includes drag force densities per unit volume generated by the relative motion of the constituents. It accounts for surface forces, but not for momentum exchange owing to transfer of inertial mass (i.e. diffusion) which is described by the term $\Pi^{\beta k} \overline{\boldsymbol{v}}_{\mathrm{I}}^{\beta k}$. Concerning locally drained fluid-saturated porous media a specific constitutive equation for $\boldsymbol{\Gamma}^{\beta k}$ will result in Darcy's law for the fluid flow. Further details about the derivation of (16) will be given in a future paper [3].

\subsection{Mixture Balance Principles}

Summation of conservation of mass $(16)_{1}$ and balance of momentum $(16)_{2}$ over all species and phases in consideration of (9) yields the corresponding macroscopic balance principles of the homogenized mixture:

$$
\begin{gathered}
\frac{\partial\langle\rho\rangle}{\partial t}+\operatorname{div}\langle\rho \boldsymbol{v}\rangle=0 \\
\frac{\partial\langle\rho \boldsymbol{v}\rangle}{\partial t}+\operatorname{div}\langle\rho \boldsymbol{v} \otimes \boldsymbol{v}\rangle=\langle\rho \boldsymbol{b}\rangle+\operatorname{div}\langle\boldsymbol{\sigma}\rangle .
\end{gathered}
$$

Here we have used the fact that the sum of the transfer terms over all constituents vanishes, that is,

$$
\sum_{k} \sum_{\beta} \Pi^{\beta k}=0 \quad \text { and } \quad \sum_{k} \sum_{\beta}\left(\Pi^{\beta k} \overline{\boldsymbol{v}}_{\mathrm{I}}^{\beta k}+\boldsymbol{\Gamma}^{\beta k}\right)=\mathbf{0}
$$

in accordance with the microscopic jump conditions (3). Only averaged quantities will be considered in the remainder of this paper.

It proofs convenient to display the balance principles derived so far in a different form. Note that each of (16) and (18) provides the so-called Eulerian conservation form of the balance principle with respect to fixed spatial points. An equivalent representation more common in solid mechanics is the (updated) Lagrangian form referring to the current configuration of the mixture in the ambient space. Conservation of mass and balance of momentum of the mixture then read

$$
\langle\dot{\rho}\rangle+\langle\rho\rangle \operatorname{div}\langle\boldsymbol{v}\rangle=0 \quad \text { and } \quad\langle\rho \dot{\boldsymbol{v}}\rangle=\langle\rho \boldsymbol{b}\rangle+\operatorname{div}\langle\boldsymbol{\sigma}\rangle
$$

respectively. Here $\langle\dot{q}\rangle$, in which $q$ is an arbitrary time-dependent spatial field, is used as an abbreviation for

$$
\langle\dot{q}\rangle \stackrel{\text { def }}{=} \sum_{k} \sum_{\beta} f^{k} \pi^{\beta k} \dot{q}^{\beta k}=\sum_{k} f^{k}\left(\left(1-n^{k}\right) \dot{q}^{\mathrm{s} k}+n^{k} \dot{q}^{\mathrm{fk}}\right),
$$


where

$$
\dot{q}^{\beta k} \stackrel{\text { def }}{=} \frac{\partial q^{\beta k}}{\partial t}+\boldsymbol{v}^{\beta k} \cdot \nabla q^{\beta k}
$$

denotes the material time derivative of a $\beta$-species- $k$-phase-related quantity following the individual motion with velocity $\boldsymbol{v}^{\beta k}$. The term $\langle\rho\rangle \operatorname{div}\langle\boldsymbol{v}\rangle$ in $(21)_{1}$ is defined as the difference between $(18)_{1}$ and $\langle\dot{\rho}\rangle$. We note that $(20)_{2}$ is equivalent to $(18)_{2}$ if and only if conservation of mass $(20)_{1}$ resp. $(18)_{1}$ is satisfied. We also remark that (22) is generally different form the material time derivative of a $k$-phase-related quantity $q^{k}$ along the $k$-phase mean velocity, which is denoted by

$$
\grave{q}^{k} \stackrel{\text { def }}{=} \frac{\partial q^{k}}{\partial t}+\boldsymbol{v}^{k} \cdot \nabla q^{k}
$$

\section{Closure of the Model}

The two equations (18) in conjunction with (19), (16), and (17) are the balance principles governing the flow of a mixture of multiple materials with several species, by including the flow of a single-phase material as a special case. Modeling the specific multi-material flow associated with vibro-injection pile installation, however, requires closure of this set of equations, which is otherwise underdetermined. Generally the following closure laws (or closure models) have to be specified $[14,15]$ :

1. Transfer laws expressing the physics at the material interfaces.

2. Constitutive laws characterizing the physical behavior of each material.

3. Topological laws accounting for the evolution of variables characterizing the interfacial structure.

Restrictions on the form of the closure laws result from the principles of constitutive theory (e.g. objectivity) and from the fact that a material phase containing fractions of both a solid species and a fluid species must represent a fluid-saturated porous medium.

\subsection{Interfacial Transfer Closure Laws}

With regard to the transfer laws for the mixture it is assumed that no momentum transfer occurs, i.e. $\boldsymbol{\Gamma}^{\beta k}=\mathbf{0}$ for all $\beta \in\{\mathrm{s}, \mathrm{f}\}$ and $k \in\left\{1, \ldots, n_{\text {mat }}\right\}$, resulting in uncoupled constituents at this stage of the derivation. Microscopic or molecular shear resistance within a constituent (e.g. grain contact forces, fluid viscosity) has to be modeled by the associated constitutive equation. Furthermore, any mass transfer, no matter between which constituents, phases, or species of the mixture, remains unconsidered such that $\Pi^{\beta k}=0$ for all $\beta \in\{\mathrm{s}, \mathrm{f}\}$ and $k \in\left\{1, \ldots, n_{\text {mat }}\right\}$. That is to say, constituents do not chemically react, no diffusion and dispersion occurs, and interfaces are impermeable. As a result the injection of grout into the pore space of the soil is not described by the model and fluid-saturated porous media in the mixture are regarded locally undrained; the drained case including 
consolidation effects will be considered in future research. We are aware that this is a drastic simplification, but is deemed necessary concerning the development from scratch of the new MMALE method outlined in Part 2.

Notwithstanding this, inflow and outflow of the homogenized mixture across non-Lagrangian boundaries of the computational domain is generally allowed. Moreover, no limitations whatsoever exist with regard to the miscibility of the water-saturated soil with grout material on the macro level, in which the material phases maintain their original properties. The underlying averaged description of the multi-material flow likewise captures separated mixtures (analogy: oil on water) as well as disperse mixtures (analogy: emulsion of oil and water).

\subsection{Constitutive Closure Laws}

The constitutive closure laws summarized next characterize the mechanical behavior of the material phases during vibro-injection pile installation (cf. Fig. 2, detail B), namely of the bulk solid phase, the bulk fluid phase, and the phase forming a fluid-saturated porous medium (sand). The objective is to determine for each phase $k$ the Cauchy stress given by

$$
\boldsymbol{\sigma}^{k}=\sum_{\beta} \pi^{\beta k} \boldsymbol{\sigma}^{\beta k}=\left(1-n^{k}\right) \boldsymbol{\sigma}^{\mathrm{sk}}+n^{k} \boldsymbol{\sigma}^{\mathrm{fk}},
$$

with the fluid fraction being either $\pi^{\mathrm{fk}}=n^{k}=0$ (bulk solid, $\boldsymbol{\sigma}^{k}=\boldsymbol{\sigma}^{\mathrm{sk}}$ ), $n^{k}=1$ (bulk fluid, $\boldsymbol{\sigma}^{k}=\boldsymbol{\sigma}^{\mathrm{f} k}$ ), or $0<n^{k}<1$ (fluid-saturated porous medium). In order to treat the mechanics of all materials of the problem (cf. Fig. 2, detail B) in a unified fashion, we recall from [39, 27] that the Cauchy stress tensor of any material can be decomposed into a pressure stress $-p^{\beta k} \boldsymbol{I}$ and an extra stress $\boldsymbol{s}^{\beta k}$, but also into a spherical part and the stress deviator $\boldsymbol{\sigma}_{\mathrm{dev}}^{\beta k} \stackrel{\text { def }}{=} \boldsymbol{\sigma}^{\beta k}-\frac{1}{3}\left(\operatorname{tr} \boldsymbol{\sigma}^{\beta k}\right) \boldsymbol{I}$ according to

$$
\boldsymbol{\sigma}^{\beta k}=-p^{\beta k} \boldsymbol{I}+\boldsymbol{s}^{\beta k}=-\bar{p}^{\beta k} \boldsymbol{I}+\boldsymbol{\sigma}_{\mathrm{dev}}^{\beta k},
$$

where $\boldsymbol{I}$ is the second-order unit tensor and $\bar{p}^{\beta k} \stackrel{\text { def }}{=}-\frac{1}{3} \operatorname{tr} \boldsymbol{\sigma}^{\beta k}$ is referred to as the (negative) mean stress. Generally one has $p^{\beta k} \neq \bar{p}^{\beta k}$ unless $\boldsymbol{s}^{\beta k}=\boldsymbol{\sigma}_{\operatorname{dev}}^{\beta k}$ resp. $\operatorname{tr} \boldsymbol{s}^{\beta k}=0$, which is usually assumed for pure solids. Fluids may possess a non-deviatoric $\boldsymbol{s}^{\beta k}$ through volume viscosity but this is not considered here; cf. $(27)_{2}$.

The decomposition of stress (25) is useful to model both compressible and nearly incompressibile materials. We then assume for phases composed of a single species (i.e. $\pi^{\beta k} \equiv 1$ ), like bulk fluid or bulk solid, that there is a compression model of the form

$$
-\left.\frac{1}{V^{\beta k}} \frac{\partial V^{\beta k}}{\partial p^{\beta k}}\right|_{M^{\beta k}}=\frac{1}{\rho^{\beta k}} \frac{\mathrm{d} \rho^{\beta k}}{\mathrm{~d} p^{\beta k}} \stackrel{\text { def }}{=} \frac{1}{K^{\beta k}} \quad \text { resp. } \quad \dot{p}^{\beta k} \stackrel{\text { def }}{=} \frac{K^{\beta k}}{\rho^{\beta k}} \dot{\rho}^{\beta k}
$$

for each constituent, relating the rate of pressure to the rate of mass density through a finite bulk modulus $K^{\beta k}$. In (26), $V^{\beta k}$ and $M^{\beta k} \stackrel{\text { def }}{=} \rho^{\beta k} V^{\beta k}$ are the 
volume and intrinsic mass of the $\beta$-species in the $k$-phase in the RVE, respectively, and $\left.\right|_{M^{\beta k}}$ means that mass is kept constant along with differentiation.

First, let us consider the bulk solid and bulk fluid phases, whose behavior is assumed to be the same at all length scales defined (micro, meso, or macro). The following constitutive assumptions are made. Effects of turbulence in the bulk fluid are currently neglected. Bulk solid is either rigid or hypoelasto-plastic, and bulk fluid is a Newtonian fluid with deviatoric viscous stress. The latter two assumptions can be formalized as

$$
\begin{aligned}
& \stackrel{\nabla}{\mathrm{s} k} \stackrel{\text { def }}{=} \boldsymbol{c}^{\mathrm{s} k}\left(\boldsymbol{\sigma}^{\mathrm{s} k}, \boldsymbol{h}^{\mathrm{s} k}\right): \boldsymbol{d}^{\mathrm{s} k} \\
& \boldsymbol{\sigma}^{\mathrm{f} k} \stackrel{\text { def }}{=}-p^{\mathrm{f} k} \boldsymbol{I}+\boldsymbol{c}^{\mathrm{f} k}: \boldsymbol{d}^{\mathrm{f} k} \quad \text { with } \quad \operatorname{tr}\left(\boldsymbol{c}^{\mathrm{f} k}: \boldsymbol{d}^{\mathrm{f} k}\right)=0,
\end{aligned}
$$

respectively, where $\boldsymbol{h} \stackrel{\text { def }}{=}\left\{h_{1}, \ldots, h_{m}\right\}$ is a set of material state variables, $\stackrel{\nabla}{\boldsymbol{\sigma}} \stackrel{\text { def }}{=}$ $\dot{\boldsymbol{\sigma}}+\boldsymbol{\sigma} \cdot \boldsymbol{\omega}-\boldsymbol{\omega} \cdot \boldsymbol{\sigma}$ denotes the Zaremba-Jaumann rate of the considered second order tensor, $\boldsymbol{\omega} \stackrel{\text { def }}{=} \frac{1}{2}\left(\nabla \boldsymbol{v}-(\nabla \boldsymbol{v})^{\mathrm{T}}\right)$ is the vorticity tensor, $\boldsymbol{d} \stackrel{\text { def }}{=} \frac{1}{2}\left(\nabla \boldsymbol{v}+(\nabla \boldsymbol{v})^{\mathrm{T}}\right)$ is the spatial rate of deformation tensor, $\operatorname{tr}(\cdot)$ returns the trace of a second-order tensor, and : indicates double contraction. The bulk fluid phase representing macroscopic void zones (e.g. ambient atmosphere) is modeled by

$$
\boldsymbol{\sigma}^{\mathrm{f} k} \stackrel{\text { def }}{=}-p^{\mathrm{f} k} \boldsymbol{I}, \quad \text { with } \quad \dot{p}^{\mathrm{f} k}=-K^{\mathrm{fk}} \operatorname{div} \boldsymbol{d}^{\mathrm{f} k} \quad \text { and } \quad K^{\mathrm{f} k} \approx 0, \rho^{\mathrm{f} k} \approx 0 .
$$

The application of (25) in conjunction with (26) to a rate constitutive equation of the form $(27)_{1}$ generally results in

$$
\stackrel{\nabla}{\boldsymbol{s}}^{\mathrm{s} k}=\stackrel{\nabla}{\mathrm{\sigma}}_{\mathrm{dev}}^{\mathrm{s} k} \stackrel{\text { def }}{=} \boldsymbol{c}^{\mathrm{s} k}\left(\boldsymbol{\sigma}^{\mathrm{s} k}, \boldsymbol{h}^{\mathrm{s} k}\right): \boldsymbol{d}_{\mathrm{dev}}^{\mathrm{s} k} \quad \text { and } \quad \dot{p}^{\mathrm{s} k} \stackrel{\text { def }}{=} \frac{\dot{\rho}^{\mathrm{s} k}}{3 \rho^{\mathrm{s} k}} \boldsymbol{I}: \boldsymbol{c}^{\mathrm{s} k}: \boldsymbol{I} .
$$

Clearly, the rate of the extra stress (rate of stress deviator) in the bulk solid can be calculated from the usual rate constitutive equation by using the deviatoric rate of deformation, and the corresponding bulk modulus can be calculated from the material tangent tensor as $3 K^{\text {sk }}=\boldsymbol{I}: \boldsymbol{c}^{\text {sk }}: \boldsymbol{I}$.

It should be noted that in a mixture of materials the material time derivative and the velocity $\boldsymbol{v}$ entering the previous relations are those related to the macroscopic motion of the actual constituent, in accordance with (22). We also remark that compressive stress is taken with negative sign, but pressure has positive sign whenever stress is compressive. This corresponds to the sign convention of general mechanics.

Two different approaches can be employed to model the mechanical behavior of sand, or porous media in general, on the mesoscale. The first approach describes the behavior on the microscale of grain size and then applies spatial averaging to obtain the behavior on the mesoscale [43]. In the second approach, which is followed here, each species is regarded as a continuum at the mesoscale defined over all space. Concerning fluid-saturated porous media in the mixture, we first notice that $\boldsymbol{v}^{\mathrm{f} k}=\boldsymbol{v}^{\mathrm{s} k}=\boldsymbol{v}^{k}$ due to the locally undrained conditions assumed. The pore fluid is taken ideal (non-viscous) such that $\boldsymbol{\sigma}^{\mathrm{f} k} \stackrel{\text { def }}{=}-p^{\mathrm{f} k} \boldsymbol{I}$, 
in contrast to $(27)_{2}$. As a consequence, the solid species velocity and pore fluid pressure are the only degrees of freedom of the undrained fluid-saturated porous medium.

Terzaghi's principle of effective stress $[45,46]$ is introduced as

$$
\boldsymbol{\sigma}^{k^{\prime}}=\boldsymbol{\sigma}^{k}+p^{\mathrm{f} k} \boldsymbol{I}
$$

where $\boldsymbol{\sigma}^{k^{\prime}}$ is Terzaghi's effective stress and $\boldsymbol{\sigma}^{k}$ is the total Cauchy stress acting on the saturated porous medium representing the $k$-phase. Using (30) in conjunction with (25), the $k$-phase mean effective stress $p^{k^{\prime}} \stackrel{\text { def }}{=}-\frac{1}{3} \operatorname{tr} \sigma^{k^{\prime}}$ can be obtained from

$$
p^{k^{\prime}}=\left(1-n^{k}\right)\left(p^{\mathrm{s} k}-p^{\mathrm{f} k}\right)=p^{k}-p^{\mathrm{f} k}, \quad \text { so that } \quad \dot{p}^{k^{\prime}}=\dot{p}^{k}-\dot{p}^{\mathrm{f} k} .
$$

Hence, the mean effective stress divided by the solid fraction equals the averaged excess pressure in the solid species, that is, $p^{k^{\prime}} /\left(1-n^{k}\right)=p^{\mathrm{s} k}-p^{\mathrm{f} k}$; in a suspension each sand grain is completely surrounded by water such that $p^{\mathrm{s} k}=p^{\mathrm{f} k}$ and $p^{k^{\prime}}=0$. The second equation in $(31)$ is the rate form of the first equation. The superposed dot indicates the material time derivative along the velocity $\boldsymbol{v}^{\mathrm{s} k}$ of the solid species in the $k$-phase (which equals the velocity of the fluid species at locally undrained conditions, see above).

The solid and fluid species of a fluid-saturated porous medium are generally compressible. This particularly means that the density of both the water and the grain material of water-saturated sand can change due to pressure loading. The mass density of the pore fluid can be considered as a function of the pore fluid pressure alone, that is, $\rho^{\mathrm{fk}}=\tilde{\rho}^{\mathrm{fk}}\left(p^{\mathrm{fk}}\right)$ such that (26) holds. The compressibility resp. the bulk modulus of pore water generally depends not only on pressure and temperature, but also on the gas content indicating partial saturation. A small air content reduces the bulk modulus of an air-water mixture considerably, hence should be considered in the calculation of the pore fluid pressure. In the present research we use the relation [25]

$$
K^{\mathrm{f} k}=\left(\frac{S^{k}}{K_{0}^{\mathrm{fk}}}+\frac{1-S^{k}}{p^{\mathrm{f} k}}\right)^{-1},
$$

where $K^{\mathrm{fk}}$ is the bulk modulus of an air-water mixture, $S^{k}$ is the degree of water saturation, $K_{0}^{\mathrm{f} k}$ is the bulk modulus of pure water $\left(S^{k}=1.0 ; K_{0}^{\mathrm{f} k}=\right.$ $4.78 \times 10^{-10} \mathrm{~Pa}^{-1}$ under atmospheric pressure at $\left.10^{\circ} \mathrm{C}\right)$, und $p^{\mathrm{f} k}$ is the absolute fluid pressure.

As opposed to the pore fluid the mass density of the solid species (grain material) in saturated porous media not only depends on the pressure but also on the porosity $[21,7,8,9]$ :

$$
\rho^{\mathrm{s} k}=\tilde{\rho}^{\mathrm{s} k}\left(p^{\mathrm{s} k}, n^{k}\right), \quad \text { or, equivalently, } \quad p^{\mathrm{sk}}=\tilde{p}^{\mathrm{sk}}\left(\rho^{\mathrm{s} k}, n^{k}\right) .
$$

Therefore, the total change in pressure within the solid species in the $k$-phase,

$$
\mathrm{d} p^{\mathrm{s} k}=\left.\frac{\partial p^{\mathrm{s} k}}{\partial \rho^{\mathrm{s} k}}\right|_{n^{k}} \mathrm{~d} \rho^{\mathrm{s} k}+\left.\frac{\partial p^{\mathrm{s} k}}{\partial n^{k}}\right|_{\rho^{\mathrm{s} k}} \mathrm{~d} n^{k},
$$


consists of a pressure change due to compression of the grains leaving the porosity unchanged and a pressure change due to rearrangement of the grains (configurational compression) at constant mass density of the grain material. In the partial derivatives the superposed ${ }^{\sim}$ is omitted for notational brevity.

Because the porosity is not directly measurable, we choose a different set of independent variables in (33) which can be combined to back out the original variables. In consideration of (31), we replace in $(33)_{1}$ the set of independent variables $\left(p^{\mathrm{s} k}, n^{k}\right)$ by the equivalent set $\left(p^{k^{\prime}}, p^{\mathrm{f} k}\right)$, so that $\rho^{\mathrm{s} k}=\tilde{\rho}^{\mathrm{s} k}\left(p^{k^{\prime}}, p^{\mathrm{f} k}\right)=$ $\tilde{\rho}^{\mathrm{s} k}\left(p^{k}-p^{\mathrm{f} k}, p^{\mathrm{fk}}\right)$. Then, the total change of volume of the $k$-phase solid species can be calculated from

$$
\begin{aligned}
-\left.\frac{\mathrm{d} V^{\mathrm{s} k}}{V^{\mathrm{s} k}}\right|_{M^{\mathrm{s} k}} & =\frac{\mathrm{d} p^{\mathrm{s} k}}{K^{\mathrm{s} k}}=\frac{\mathrm{d} \rho^{\mathrm{s} k}}{\rho^{\mathrm{s} k}}=\left.\frac{1}{\rho^{\mathrm{s} k}} \frac{\partial \rho^{\mathrm{s} k}}{\partial\left(p^{k}-p^{\mathrm{f} k}\right)}\right|_{p^{\mathrm{f} k}} \mathrm{~d} p^{k^{\prime}}+\left.\frac{1}{\rho^{\mathrm{s} k}} \frac{\partial \rho^{\mathrm{s} k}}{\partial p^{\mathrm{f} k}}\right|_{p^{k^{\prime}}} \mathrm{d} p^{\mathrm{f} k} \\
& =\left.\frac{1}{\rho^{\mathrm{s} k}} \frac{\partial \rho^{\mathrm{s} k}}{\partial p^{k}}\right|_{p^{\mathrm{f} k}} \mathrm{~d} p^{k^{\prime}}+\left.\frac{1}{\rho^{\mathrm{s} k}} \frac{\partial \rho^{\mathrm{s} k}}{\partial p^{\mathrm{f} k}}\right|_{p^{k^{\prime}}} \mathrm{d} p^{\mathrm{f} k}=\frac{\mathrm{d} p^{k^{\prime}}}{K_{\mathrm{d}}^{k}}+\frac{\mathrm{d} p^{\mathrm{f} k}}{K_{\mathrm{uj}}^{k}}
\end{aligned}
$$

$K^{\mathrm{s} k}$ is the material bulk modulus of the solid species, which is assumed to be a function of the mass density $\rho^{\mathrm{s} k}$ alone so that (26) applies. Note that in general, the solid species bulk modulus is also a function of the porosity. The bulk moduli $K_{\mathrm{d}}^{k}$ and $K_{\mathrm{uj}}^{k}$ have likewise been introduced in accordance with (26) and are referred to as the drained bulk modulus and unjacketed bulk modulus of the $k$-phase porous medium, respectively [8]. $K_{\mathrm{uj}}^{k}$ is approximately equal to $K^{\mathrm{s} k}$ under the assumption that the solid species volume changes only little during an unjacketed test. $K_{\mathrm{d}}^{k}$ is the bulk modulus of the drained porous medium as measured in a jacketed test.

By replacing in (35) the total differential with the material time derivative along the velocity $\boldsymbol{v}^{\mathrm{sk}}$, and noting that $\dot{V}^{\mathrm{s} k} /\left.V^{\mathrm{s} k}\right|_{M^{\mathrm{s} k}}=\operatorname{div} \boldsymbol{v}^{\mathrm{s} k}$, one obtains

$$
\dot{p}^{k^{\prime}}=-K_{\mathrm{d}}^{k}\left(\operatorname{div} \boldsymbol{v}^{\mathrm{s} k}+\frac{\dot{p}^{\mathrm{fk}}}{K_{\mathrm{uj}}^{k}}\right)=\dot{p}^{k^{\prime \prime}}-\frac{K_{\mathrm{d}}^{k}}{K_{\mathrm{uj}}^{k}} \dot{p}^{\mathrm{f} k} .
$$

where $\dot{p}^{k^{\prime \prime}} \stackrel{\text { def }}{=}-K_{\mathrm{d}}^{k} \operatorname{div} \boldsymbol{v}^{\mathrm{sk}}$. Using $(31)_{2}$ and $K_{\mathrm{uj}}^{k} \approx K^{\mathrm{s} k}$ one arrives at

$$
\dot{p}^{k}=\dot{p}^{k^{\prime \prime}}+\alpha^{k} \dot{p}^{\mathrm{f} k}, \quad \text { where } \quad \alpha^{k} \stackrel{\text { def }}{=} 1-\frac{K_{\mathrm{d}}^{k}}{K^{\mathrm{s} k}} .
$$

The coefficient $\alpha^{k}$ is due to $[12,13]$ and accounts for the compressibility of the solid material forming the porous medium. A common approximation for sand is $\alpha^{k}=1$, meaning that the grain material is incompressible. A suspension of sand and water is characterized by $K_{\mathrm{d}}^{k}=0$, leading to $\alpha^{k}=1$ likewise. From (36) together with $\dot{p}^{\text {sk }} / K^{\text {sk }}=-\operatorname{div} \boldsymbol{v}^{\text {sk }}$ we also have

$$
\dot{p}^{\mathrm{s} k}=\dot{p}^{k^{\prime}} \frac{K^{\mathrm{s} k}}{K_{\mathrm{d}}^{k}}+\dot{p}^{\mathrm{f} k} .
$$

We now turn to the mechanical behavior of sand under general deformations. In the present work, sand is modeled as a hypoplastic porous medium, in accordance with Subproject 1 of the DFG Research Unit FOR 1136. A widely-used 
hypoplastic model for sand neglecting viscous effects is due to [41] and has been extended by [33]. Its general form,

$$
\stackrel{\nabla}{\boldsymbol{\sigma}^{\prime}} \stackrel{\text { def }}{=} \boldsymbol{c}^{k^{\prime}}\left(\boldsymbol{\sigma}^{k^{\prime}}, \boldsymbol{h}^{k^{\prime}}\right):\left(\boldsymbol{d}^{\mathrm{s} k}-\dot{\varepsilon}_{\mathrm{vol}}^{\mathrm{s}} \boldsymbol{I}\right) \stackrel{\text { def }}{=} \stackrel{\nabla}{\boldsymbol{\sigma}^{k^{\prime \prime}}}-\dot{\varepsilon}_{\mathrm{vol}}^{\mathrm{s}} \boldsymbol{c}^{k}: \boldsymbol{I},
$$

provides a spatial rate constitutive equation for the effective Cauchy stress, but here we subtracted from $\boldsymbol{d}^{\text {sk }}$ the average volumetric strain rate $\dot{\varepsilon}_{\mathrm{vol}}^{\mathrm{s}} \boldsymbol{I}$ of the solid grains under the assumption of a compressible solid species. The stress tensor $\boldsymbol{\sigma}^{k^{\prime \prime}}$ whose Zaremba-Jaumann rate is defined in (39) is responsible for all deformation of the solid species in the $k$-phase. The particular form of $\boldsymbol{c}^{k^{\prime}}\left(\boldsymbol{\sigma}^{k^{\prime}}, \boldsymbol{h}^{k^{\prime}}\right)$ can be found in the references and is assumed to be valid for dry, fully-saturated drained, and locally undrained sand. Moreover, we postulate $\boldsymbol{c}^{k^{\prime}}\left(\boldsymbol{\sigma}^{k^{\prime}}, \boldsymbol{h}^{k^{\prime}}\right) \equiv$ $\boldsymbol{c}^{k^{\prime \prime}}\left(\boldsymbol{\sigma}^{k^{\prime \prime}}, \boldsymbol{h}^{k^{\prime \prime}}\right)$ because in soil mechanics the grain material is usually taken incompressible.

A basic property of the Zaremba-Jaumann rate is $\operatorname{tr} \stackrel{\nabla}{\boldsymbol{\sigma}}=\boldsymbol{I}: \stackrel{\nabla}{\boldsymbol{\sigma}}=\boldsymbol{I}: \dot{\boldsymbol{\sigma}}[5$, p. 139], so that the trace of (39) yields

$$
\dot{p}^{k^{\prime}}=\dot{p}^{k^{\prime \prime}}+\frac{\dot{\varepsilon}_{\mathrm{vol}}^{\mathrm{s}}}{3} \boldsymbol{I}: \boldsymbol{c}^{k}: \boldsymbol{I},
$$

where $\dot{p}^{k^{\prime \prime}} \stackrel{\text { def }}{=}-\frac{1}{3} \operatorname{tr} \dot{\boldsymbol{\sigma}}^{k^{\prime \prime}}=-\frac{1}{3} \operatorname{tr}\left(\boldsymbol{c}^{k}: \boldsymbol{d}^{\text {sk }}\right)$. Comparison with (36) shows that

$$
\dot{\varepsilon}_{\mathrm{vol}}^{\mathrm{s}}=\frac{\dot{p}^{\mathrm{f} k}}{K_{\mathrm{uj}}^{k}} \approx \frac{\dot{p}^{\mathrm{f} k}}{K^{\mathrm{s} k}} \quad \text { and } \quad K_{\mathrm{d}}^{k}=\frac{\boldsymbol{I}: \boldsymbol{c}^{k}: \boldsymbol{I}}{3} .
$$

Here $\boldsymbol{c}^{k}$ is the hypoplastic material tangent of (39), but it can be any other material tangent representing generally non-isotropic porous media.

In (39) the generally unsymmetric tensor $\boldsymbol{c}^{k}: \boldsymbol{I}$ is replaced with its average $K_{\mathrm{d}}^{k} \boldsymbol{I}$ derived from $(41)_{2}[45]$. Then, by taking the Zaremba-Jaumann rate of (30),

$$
\stackrel{\nabla}{\boldsymbol{\sigma}^{k}}=\stackrel{\nabla}{\boldsymbol{\sigma}}^{k^{\prime \prime}}-\alpha^{k} \dot{p}^{\mathrm{f} k} \boldsymbol{I} .
$$

Integrating this equation by starting from a zero initial state $\left(\left.\boldsymbol{\sigma}^{k^{\prime \prime}}\right|_{t=0}=\mathbf{0}\right.$ and $\left.\left.p^{\mathrm{f} k}\right|_{t=0}=0\right)$ yields the modified principle of effective stress $[12,45,46]$

$$
\boldsymbol{\sigma}^{k^{\prime \prime}}=\boldsymbol{\sigma}^{k}+\alpha^{k} p^{\mathrm{f} k} \boldsymbol{I} .
$$

We will use this principle instead of (30) in what follows despite the fact that, strictly speaking, (43) in contrast to (30) holds only if the porous medium is characterized by linear isotropic behavior. However, for sand the coefficient $\alpha^{k}$ is indeed very close to unity.

Based on (25) and (43), it can be shown that the effective stress is related to the pressures and stresses in the constituents of the saturated porous media through

$$
\begin{aligned}
\boldsymbol{\sigma}^{k^{\prime \prime}} & =\left(1-n^{k}\right) \boldsymbol{\sigma}^{\mathrm{s} k}+\left(\alpha^{k}-n^{k}\right) p^{\mathrm{f} k} \boldsymbol{I} \\
& =-\left(\left(1-n^{k}\right) p^{\mathrm{s} k}-\left(\alpha^{k}-n^{k}\right) p^{\mathrm{f} k}\right) \boldsymbol{I}+\left(1-n^{k}\right) \boldsymbol{s}^{\mathrm{s} k},
\end{aligned}
$$




$$
\begin{gathered}
\text { with } \boldsymbol{s}^{\mathrm{sk}}=\boldsymbol{\sigma}_{\mathrm{dev}}^{\mathrm{sk}}=\boldsymbol{\sigma}_{\mathrm{dev}}^{k^{\prime \prime}} /(1-n)=\boldsymbol{s}^{k^{\prime \prime}} /(1-n)=\boldsymbol{s}^{k} /(1-n), \text { and } \\
\boldsymbol{\nabla}^{k^{\prime \prime}}=\boldsymbol{\sigma}_{\mathrm{dev}}^{k^{\prime \prime}}=\boldsymbol{c}^{k}\left(\boldsymbol{\sigma}^{k^{\prime \prime}}, \boldsymbol{h}^{k^{\prime \prime}}\right): \boldsymbol{d}_{\mathrm{dev}}^{\mathrm{sk}}
\end{gathered}
$$

by (39). Since locally undrained conditions have been assumed, the rate of pore fluid pressure can be determined from the deformation of the porous medium as $[45,46]$

$$
\dot{p}^{\mathrm{fk}}=-\alpha^{k} Q^{k} \operatorname{div} \boldsymbol{v}^{\mathrm{sk}}, \quad \text { with } \quad \frac{1}{Q^{k}} \stackrel{\text { def }}{=} \frac{\alpha^{k}-n^{k}}{K^{\mathrm{s} k}}+\frac{n^{k}}{K^{\mathrm{fk}}},
$$

This relation can be derived using conservation of mass for each species. Therefore,

$$
\dot{p}^{k}=-K^{k} \operatorname{div} \boldsymbol{v}^{\mathrm{s} k}
$$

by (37), in which

$$
K^{k}=K_{\mathrm{d}}^{k}\left(1+\frac{\left(\alpha^{k}\right)^{2}}{\alpha^{k} K_{\mathrm{d}}^{k} / K^{\mathrm{s} k}+n^{k}\left(K_{\mathrm{d}}^{k} / K^{\mathrm{f} k}-K_{\mathrm{d}}^{k} / K^{\mathrm{s} k}\right)}\right)
$$

represents, in the most general case, the bulk modulus of an undrained saturated porous material with compressible constituents [19]. The remaining cases are:

- Bulk solid phase $\left(n^{k}=0, K_{d}^{k}=K^{\mathrm{s} k}, \alpha^{k}=0\right)$, for which $K^{k}=K^{\mathrm{s} k}$.

- Bulk fluid phase $\left(n^{k}=1, K_{\mathrm{d}}^{k}=0, \alpha^{k}=1\right)$, for which $K^{k}=K^{\mathrm{f} k}$.

- Dry porous medium $\left(0<n^{k}<1, K^{\mathrm{f} k} \approx 0\right)$, for which $K^{k}=K_{\mathrm{d}}^{k}$.

- Suspension of sand and water $\left(K_{\mathrm{d}}^{k}=0, \alpha^{k}=1\right)$, for which $p^{\mathrm{s} k}=p^{\mathrm{fk}}$ and $K^{k}=\left(\left(1-n^{k}\right) / K^{\mathrm{s} k}+n^{k} / K^{\mathrm{f} k}\right)^{-1}$.

In concluding this section, we remark that the velocity $\boldsymbol{v}^{k}$ and pressure $p^{k}$ of each phase $k$ represent its degrees of freedom (primary unknowns). Each phase can be a solid, a fluid, or a fluid-saturated porous medium depending on the fluid fraction $n^{k}$ assigned at the outset. Accordingly, the developed model is able to describe the flow and large deformation of mixtures of solids, fluids, and porous media in a unified fashion.

\subsection{Topological Closure Laws}

The application of spatial averaging to multi-material flows entails a loss of information as it smoothes out details of the flow structure, like the geometry of the material interfaces $[14,15]$. The topological closure laws should restore the lost information. Because the flow structure results from quantities related to the problem as a whole and not from intrinsic material properties alone, topological closure laws are not closure laws in a strict sense. Concerning the multi-material flow associated with vibro-injection pile installation and the assumptions and restrictions made so far, the only topological laws required are those that account for the evolution of the $k$-phase volume fraction $f^{k}$ and fluid fraction $n^{k}$. To show this, we analyze the governing equations (16) subject to the constraints (9). 
The external loads $\boldsymbol{b}^{\mathrm{s} k}$ and $\boldsymbol{b}^{\mathrm{f} k}$ are assumed to be given, and the unknowns for each phase $k$ are the mass densities $\rho^{\mathrm{s} k}$ and $\rho^{\mathrm{f} k}$, the velocities $\boldsymbol{v}^{\mathrm{s} k}$ and $\boldsymbol{v}^{\mathrm{f} k}$, the stresses $\boldsymbol{\sigma}^{\mathrm{s} k}$ and $\boldsymbol{\sigma}^{\mathrm{f} k}$, the volume fraction $f^{k}$, the fractions of the solid and fluid species $\pi^{\mathrm{s} k}$ and $\pi^{\mathrm{f} k}$, respectively, the mass transfer terms $\Pi^{\mathrm{s} k}$ and $\Pi^{\mathrm{f} k}$, as well as the momentum transfer terms $\boldsymbol{\Gamma}^{\mathrm{s} k}$ and $\boldsymbol{\Gamma}^{\mathrm{f} k}$. Mass and momentum transfer was assumed zero, and the constraint $(9)_{2}$ yields $\pi^{\mathrm{s} k}=1-\pi^{\mathrm{f} k}=1-n^{k}$. Since the stress tensors are symmetric one is left with a total of $22 n_{\text {mat }}$ unknowns in threedimensional space: $2 n_{\text {mat }}$ mass densities (resp. pressures, by (26)), $6 n_{\text {mat }}$ velocity components, $12 n_{\text {mat }}$ stress components, $n_{\text {mat }}$ volume fractions, and $n_{\text {mat }}$ fluid fractions (porosities). These unknowns are accompanied by the $2 n_{\text {mat }}$ equations of conservation of mass and $6 n_{\text {mat }}$ equations of balance of momentum for both species in all material phases, and by the constraint $(9)_{1}$. The balance principles of the mixture (18) do not provide additional information, but for the $12 n_{\text {mat }}$ stress components of $\boldsymbol{\sigma}^{\mathrm{s} k}$ and $\boldsymbol{\sigma}^{\mathrm{fk}}$, respectively of combinations of these, the constitutive equations of the previous section are substituted. Therefore, the number of unknowns is finally reduced to $2 n_{\text {mat }}-1$, namely $n_{\text {mat }}-1$ volume fractions and $n_{\text {mat }}$ porosities.

The basic equations to tackle this problem of closure are the constraints (9) as well as the mass conservation equation of the $\beta$-species of the $k$-phase $(16)_{1}$, with $\beta \in\{\mathrm{s}, \mathrm{f}\}$ and $k \in\left\{1, \ldots, n_{\text {mat }}\right\}$, under the assumption of zero mass transfer between the constituents. First, we notice that time derivation of the constraints (9) and the application of a basic averaging rule [20] results in

$$
\sum_{k} \frac{\partial f^{k}}{\partial t}=0 \quad \text { and } \quad \sum_{\beta} \frac{\partial \pi^{\beta k}}{\partial t}=0
$$

The second condition is automatically satisfied since $\pi^{\mathrm{s} k}=1-\pi^{\mathrm{f} k}$. Moreover, due to the fact that $\boldsymbol{v}^{\mathrm{f} k}=\boldsymbol{v}^{\mathrm{s} k}=\boldsymbol{v}^{k}$ under the assumption of locally undrained conditions the $n_{\text {mat }}$ unknown porosities can be determined. This is done by expanding $(16)_{1}$ for the case where $n_{\text {mat }}=1$, so that $f^{k}=1$ :

$$
\frac{\partial \pi^{\beta k} \rho^{\beta k}}{\partial t}+\operatorname{div}\left(\pi^{\beta k} \rho^{\beta k} \boldsymbol{v}^{\beta k}\right)=\rho^{\beta k} \dot{\pi}^{\beta k}+\pi^{\beta k} \dot{\rho}^{\beta k}+\pi^{\beta k} \rho^{\beta k} \operatorname{div} \boldsymbol{v}^{\beta k}=0
$$

Hence, the porosity $\pi^{\mathrm{f} k}=n^{k}$ is updated by

$$
\dot{n}^{k}=\left(1-n^{k}\right)\left(\frac{\dot{\rho}^{\mathrm{s} k}}{\rho^{\mathrm{s} k}}+\operatorname{div} \boldsymbol{v}^{\mathrm{s} k}\right)=\left(1-n^{k}\right)\left(\frac{\dot{p}^{\mathrm{s} k}}{K^{\mathrm{s} k}}+\operatorname{div} \boldsymbol{v}^{\mathrm{s} k}\right)
$$


Next, we consider $(16)_{1}$ for arbitrary $n_{\text {mat }}>1$ but we take the sum over all species in consideration of $(9)_{2}$ :

$$
\begin{aligned}
0 & =\sum_{\beta} \frac{\partial f^{k} \pi^{\beta k} \rho^{\beta k}}{\partial t}+\sum_{\beta} \operatorname{div}\left(f^{k} \pi^{\beta k} \rho^{\beta k} \boldsymbol{v}^{\beta k}\right) \\
& =\frac{\partial}{\partial t} \sum_{\beta} f^{k} \pi^{\beta k} \rho^{\beta k}+\operatorname{div} \sum_{\beta} f^{k} \pi^{\beta k} \rho^{\beta k} \boldsymbol{v}^{\beta k}=\frac{\partial f^{k} \rho^{k}}{\partial t}+\operatorname{div}\left(f^{k} \rho^{k} \boldsymbol{v}^{k}\right) \\
& =\rho^{k}\left(\frac{\partial f^{k}}{\partial t}+\mathbf{d} f^{k} \cdot \boldsymbol{v}^{k}\right)+f^{k}\left(\frac{\partial \rho^{k}}{\partial t}+\operatorname{div}\left(\rho^{k} \boldsymbol{v}^{k}\right)\right) \\
& =\rho^{k} \grave{f}^{k}+f^{k} \grave{\rho}^{k}+f^{k} \rho^{k} \operatorname{div} \boldsymbol{v}^{k},
\end{aligned}
$$

where $\mathbf{d}(\cdot)$ returns the differential (or gradient) of a scalar-valued function. That is,

$$
\grave{f}^{k}=-f^{k}\left(\frac{\grave{\rho}^{k}}{\rho^{k}}+\operatorname{div} \boldsymbol{v}^{k}\right) .
$$

The equality of the first and second lines in (52) can again be shown by basic averaging rules [20], and the material time derivative $\grave{q}^{k}$ of a $k$-phase-related quantity $q^{k}$ has been defined through (23).

It is crucial to note that (53) neither yields additional information nor defines a topological closure law for the volume fraction. Equation (53) is just a rearranged form of conservation of mass with regard to the $k$-phase of the mixture. A proper closure law for volume fraction instead has to account for the physics of the problem and particularly has to specify how the volumetric distribution of the bulk solid, the bulk fluid, and the saturated porous medium evolves during vibro-injection pile installation. Further research is needed to establish such a topological closure law.

\subsection{Resulting Model for Multi-Material Flow}

The derivations presented so far result in a macroscopic model for the mechanics of the multi-material flow associated with vibro-injection pile installation in saturated sand:

$$
\begin{aligned}
\operatorname{div}\langle\boldsymbol{s}-p \boldsymbol{I}\rangle+\langle\rho \boldsymbol{b}\rangle-\langle\rho \dot{\boldsymbol{v}}\rangle & =\mathbf{0} \\
\left\langle\frac{\dot{p}}{K}\right\rangle+\operatorname{div}\langle\boldsymbol{v}\rangle & =0,
\end{aligned}
$$


where

$$
\begin{array}{r}
\langle\boldsymbol{s}\rangle=\sum_{k} f^{k} \boldsymbol{s}^{k}=\sum_{k} f^{k}\left(\left(1-n^{k}\right) \boldsymbol{s}^{\mathrm{s} k}+n^{k} \boldsymbol{s}^{\mathrm{f} k}\right)=\sum_{k} f^{k} \boldsymbol{s}^{k^{\prime \prime}}, \\
\langle p\rangle=\sum_{k} f^{k} p^{k}=\sum_{k} f^{k}\left(\left(1-n^{k}\right) p^{\mathrm{s} k}+n^{k} p^{\mathrm{f} k}\right)=\sum_{k} f^{k}\left(p^{k^{\prime \prime}}+\alpha^{k} p^{\mathrm{f} k}\right), \\
\langle\rho\rangle=\sum_{k} f^{k} \rho^{k}=\sum_{k} f^{k}\left(\left(1-n^{k}\right) \rho^{\mathrm{s} k}+n^{k} \rho^{\mathrm{f} k}\right), \quad\langle\rho \dot{\boldsymbol{v}}\rangle=\sum_{k} f^{k} \rho^{k} \grave{\boldsymbol{v}}^{k}, \\
\left\langle\frac{\dot{p}}{K}\right\rangle=\sum_{k} \frac{f^{k}}{K^{k}} \grave{p}^{k}, \quad \operatorname{div}\langle\boldsymbol{v}\rangle=\sum_{k}\left(\grave{f}^{k}+f^{k} \operatorname{div} \boldsymbol{v}^{k}\right), \\
K^{k}=K_{\mathrm{d}}^{k}\left(1+\frac{\left(\alpha^{k}\right)^{2}}{\alpha^{k} K_{\mathrm{d}}^{k} / K^{\mathrm{s} k}+n^{k}\left(K_{\mathrm{d}}^{k} / K^{\mathrm{f} k}-K_{\mathrm{d}}^{k} / K^{\mathrm{s} k}\right)}\right), \quad \alpha^{k}=1-\frac{K_{\mathrm{d}}^{k}}{K^{\mathrm{s} k}} .
\end{array}
$$

This model, in conjunction with (19), (16), and (17), is closed by the constitutive equations for the bulk solid, bulk fluid, porous medium, pore fluid, and void, and by the evolution equations (topological closure laws) for the porosities $n^{k}$ and volume fractions $f^{k}$. The necessary topological closure laws for the $n_{\text {mat }}-1$ volume fractions are yet unspecified. It will be shown in the next section, however, that a priori closure respecting the assumptions underlying the applicability of an MMALE method give rise to proper relations.

\section{Two-Equation Reduced Model}

\subsection{A Priori Closure (Subcell Model)}

In addition to the closure laws related to the continuous problem addressed in Sect. 4, the application of an MMALE numerical method calls for a special closure model for multi-material elements, referred to as the subcell model, in order to render the discretized problem well-posed $[10,11,17,29,36,30]$. This subcell model solves the problem of relating the evolution of the individual materials in multi-material elements to the macroscopic degrees of freedom of the element. In developing the subcell model we keep things as simple as possible and a priori assume homogeneous distributions of pressure and velocity in the mixture at each instant of time:

$$
p^{k}=\langle p\rangle \quad \text { and } \quad \boldsymbol{v}^{k}=\langle\boldsymbol{v}\rangle \quad \text { for all } k \in\left\{1, \ldots, n_{\text {mat }}\right\} \text { and } t \in[0, T] .
$$

A direct consequence of these two assumptions is that each element also has a single deviatoric strain rate, that is, $\boldsymbol{d}_{\mathrm{dev}}^{k}=\left\langle\boldsymbol{d}_{\mathrm{dev}}\right\rangle$ for all $k \in\left\{1, \ldots, n_{\mathrm{mat}}\right\}$.

From a physical viewpoint, (56) means that everything is in homogeneous thermodynamic equilibrium [37, 17, 29]. The assumption of pressure equilibrium is reasonable because pressure is continuous across a material interface. Pressure disequilibration is associated with highly-dynamic compaction or other processes not considered here. If $(56)_{1}$ holds, then the adjustment of volume 
fractions and the transfer of pressure and pressure changes is infinitely fast. The requirement that each element has a single velocity, on the other hand, is not appropriate because equilibration of velocity differences is driven by drag forces on material interfaces (momentum transfer). Assumption $(56)_{2}$ results in fullybonded material phases without a contact mechanism. However, it is commonly accepted because tangential contact with or without friction is difficult to model in MMALE and multi-material Eulerian methods [40]. As a consequence, shear resistance is accounted for only by the constitutive equation inside of the phases next to the interface.

Based on the assumptions (56), the continuum mechanical two-equation model summarized in Sect. 4.4 can now be reduced; see also [17, 29]. In particular, (53) under the assumption $(56)_{2}$ becomes

$$
\dot{f}^{k}+f^{k} \operatorname{div}\langle\boldsymbol{v}\rangle=-\frac{\dot{\rho}^{k}}{\rho^{k}}=-f^{k} \frac{\dot{p}^{k}}{K^{k}},
$$

in which the superposed dot now represents the material time derivative of any spatial field $q$ along the mean velocity $\langle\boldsymbol{v}\rangle$ of the mixture:

$$
\dot{q} \stackrel{\text { def }}{=} \frac{\partial q}{\partial t}+\langle\boldsymbol{v}\rangle \cdot \nabla q \text {. }
$$

The assumption $(56)_{1}$ yields

$$
\left\langle\frac{\dot{p}}{K}\right\rangle=\sum_{k} \frac{f^{k} \dot{p}^{k}}{K^{k}}=\frac{\langle\dot{p}\rangle}{\langle K\rangle}, \quad \text { with } \quad \frac{1}{\langle K\rangle}=\sum_{k} \frac{f^{k}}{K^{k}} .
$$

Moreover,

$$
\frac{K^{k}}{\rho^{k}} \dot{\rho}^{k}=\dot{p}^{k}=\langle\dot{p}\rangle=\left\langle\frac{K}{\rho} \dot{\rho}\right\rangle=\langle K\rangle\left\langle\frac{\dot{\rho}}{\rho}\right\rangle,
$$

so that (57) in conjunction with conservation of mass of the mixture, $\langle\dot{\rho}\rangle+$ $\langle\rho\rangle \operatorname{div}\langle\boldsymbol{v}\rangle=0$, results in the self-consistent balance equation

$$
\dot{f}^{k}+f^{k} \operatorname{div}\langle\boldsymbol{v}\rangle=-f^{k} \frac{\langle K\rangle}{K^{k}}\left\langle\frac{\dot{\rho}}{\rho}\right\rangle=f^{k} \frac{\langle K\rangle}{K^{k}} \operatorname{div}\langle\boldsymbol{v}\rangle,
$$

that is,

$$
\dot{f}^{k}=f^{k}\left(\frac{\langle K\rangle}{K^{k}}-1\right) \operatorname{div}\langle\boldsymbol{v}\rangle .
$$

This is the remaining topological closure law for the volume fraction. Note that it naturally provides for a void collapse mechanism because the material with the smallest bulk modulus contributes most to the total volume change. This feature is of particular importance in cases where the compressibilities of the materials phases are widely different, as for example in a mixture of void and steel.

Because of (60) and the basic constraint $\sum_{k} f^{k}=1$, summation of (62) over the $n_{\text {mat }}$ phases in the mixture results in (59). Therefore, the topological closure law also satisfies the constraint $\sum_{k} \dot{f}^{k}=0$. 


\subsection{Application to the Developed Model}

The macroscopic model for the multi-material flow associated with vibro-injection pile installation summarized in Sect. 4.4 and the subcell model developed in the previous section result in following two-equation (two-field) reduced model:

$$
\begin{aligned}
\operatorname{div}\langle\boldsymbol{s}-p \boldsymbol{I}\rangle+\langle\rho \boldsymbol{b}\rangle-\langle\rho\rangle\langle\dot{\boldsymbol{v}}\rangle & =\mathbf{0} \\
\langle\dot{p}\rangle /\langle K\rangle+\operatorname{div}\langle\boldsymbol{v}\rangle & =0
\end{aligned}
$$

where

$$
\begin{array}{r}
\langle\boldsymbol{s}\rangle=\sum_{k} f^{k} \boldsymbol{s}^{k}=\sum_{k} f^{k}\left(\left(1-n^{k}\right) \boldsymbol{s}^{\mathrm{s} k}+n^{k} \boldsymbol{s}^{\mathrm{f} k}\right)=\sum_{k} f^{k} \boldsymbol{s}^{k^{\prime \prime}} \\
\langle p\rangle=\sum_{k} f^{k} p^{k}=\sum_{k} f^{k}\left(\left(1-n^{k}\right) p^{\mathrm{s} k}+n^{k} p^{\mathrm{f} k}\right)=\sum_{k} f^{k}\left(p^{k^{\prime \prime}}+\alpha^{k} p^{\mathrm{f} k}\right), \\
\langle\rho\rangle=\sum_{k} f^{k} \rho^{k}=\sum_{k} f^{k}\left(\left(1-n^{k}\right) \rho^{\mathrm{s} k}+n^{k} \rho^{\mathrm{f} k}\right), \\
\langle K\rangle^{-1}=\sum_{k} f^{k} / K^{k}, \quad \alpha^{k}=1-K_{\mathrm{d}}^{k} / K^{\mathrm{s} k}, \\
\text { and } K^{k}=K_{\mathrm{d}}^{k}\left(1+\frac{\left(\alpha^{k}\right)^{2}}{\alpha^{k} K_{\mathrm{d}}^{k} / K^{\mathrm{s} k}+n^{k}\left(K_{\mathrm{d}}^{k} / K^{\mathrm{f} k}-K_{\mathrm{d}}^{k} / K^{\mathrm{s} k}\right)}\right) .
\end{array}
$$

The model is closed by the general constitutive equations for the

bulk solid: $\quad \stackrel{\nabla}{\boldsymbol{\sigma}}^{\mathrm{s} k}=\boldsymbol{c}^{\mathrm{s} k}\left(\boldsymbol{\sigma}^{\mathrm{s} k}, \boldsymbol{h}^{\mathrm{s} k}\right):\langle\boldsymbol{d}\rangle$,

bulk fluid: $\quad \boldsymbol{\sigma}^{\mathrm{f} k}=-p^{\mathrm{f} k} \boldsymbol{I}+\boldsymbol{c}^{\mathrm{f} k}:\langle\boldsymbol{d}\rangle \quad$ subject to

$$
\operatorname{tr}\left(\boldsymbol{c}^{\mathrm{f} k}:\langle\boldsymbol{d}\rangle\right)=0 \quad \text { and } \quad \dot{p}^{\mathrm{fk}}=-K^{\mathrm{fk}} \operatorname{div}\langle\boldsymbol{d}\rangle,
$$

porous medium: $\quad \stackrel{\nabla}{k^{\prime \prime}}=\boldsymbol{c}^{k^{\prime \prime}}\left(\boldsymbol{\sigma}^{k^{\prime \prime}}, \boldsymbol{h}^{k^{\prime \prime}}\right):\langle\boldsymbol{d}\rangle$,

pore fluid: $\boldsymbol{\sigma}^{\mathrm{f} k}=-p^{\mathrm{f} k} \boldsymbol{I}$ subject to

$$
\dot{p}^{\mathrm{f} k}=-\alpha^{k} Q^{k} \operatorname{div}\langle\boldsymbol{d}\rangle \text { and } \quad \frac{1}{Q^{k}}=\frac{\alpha^{k}-n^{k}}{K^{\mathrm{s} k}}+\frac{n^{k}}{K^{\mathrm{f} k}},
$$

void: $\quad \boldsymbol{\sigma}^{\mathrm{f} k}=-p^{\mathrm{f} k} \boldsymbol{I} \quad$ subject to

$$
\dot{p}^{\mathrm{f} k}=-K^{\mathrm{f} k} \operatorname{div}\langle\boldsymbol{d}\rangle \text { and } K^{\mathrm{f} k} \approx 0, \rho^{\mathrm{f} k} \approx 0,
$$

and by the evolution equations for the porosities,

$$
\dot{n}^{k}=\left(1-n^{k}\right)\left(\frac{\dot{p}^{\mathrm{s} k}}{K^{\mathrm{s} k}}+\operatorname{div}\langle\boldsymbol{v}\rangle\right), \quad \text { where } \quad \dot{p}^{\mathrm{s} k}=\dot{p}^{k^{\prime}} \frac{K^{\mathrm{s} k}}{K_{\mathrm{d}}^{k}}+\dot{p}^{\mathrm{f} k},
$$

and volume fractions,

$$
\dot{f}^{k}=f^{k}\left(\frac{\langle K\rangle}{K^{k}}-1\right) \operatorname{div}\langle\boldsymbol{v}\rangle
$$


Finally, the mass density of the mixture is updated by $\langle\dot{\rho}\rangle=\sum_{k} f^{k} \dot{\rho}^{k}$ using the $k$-phase compression model

$$
\dot{\rho}^{k}=\frac{\rho^{k}}{K^{k}}\langle\dot{p}\rangle .
$$

We remark that the reduced model might be derived by an asymptotic analysis of the unreduced model in the limit of zero relaxation (equilibration) times instead of using a priori closure through (56) [24, 31].

\section{Conclusions and Outlook}

We have derived a continuum mechanical model to describe the multi-material flow associated with vibro-injection pile installation in saturated sand. The model has been derived from microscopic balance principles through spatial averaging and treats the mixture of multiple materials as an effective single-phase material or homogenized mixture on the macroscale. In doing so, we have assumed that each phase of the mixture is composed of a solid species and a fluid species, with the portion of the fluid being zero in a pure solid phase (bulk solid) and the portion of the solid being zero in a pure fluid phase (bulk fluid). In general, each phase represents a solid-fluid compound in which the solid species is constituted of grains of a granular material; sand in the present case. The solid-fluid compound might thus represent a fluid-saturated porous medium or a suspension. Each constituent is assumed compressible.

An important step in the development of the macroscopic continuum mechanical model has been the closure of the underlying set of equations in such a way that the specific multi-material flow associated with vibro-injection pile installation in saturated sand is described. General closure models have been defined in order to account for the physics of each material and at the material interfaces. In particular, the macroscopic mechanical behavior of a porous medium representing sand is described by a hypoplastic rate constitutive equation advanced in the Subproject 1 of this research unit. Closure models are also required for the evolution of variables characterizing the interfacial structure. These latter models, called topological closure laws, had been initially left uncompleted because no evolution equation could be specified for the volume fractions of the phases in the mixture. By assuming a priori homogeneous distributions of pressure and velocity for all phases of the mixture the set of equations have finally been closed, resulting in a two-equation (two-field) reduced model. This model will be employed in Part 2 to develop a new multi-material arbitrary Lagrangian-Eulerian (MMALE) numerical method particularly suitable to simulate pile installation.

Acknowledgments. The presented work was carried out under the financial support from the German Research Foundation (DFG; grants SA 310/26-1 and SA 310/26-2) as part of the DFG Research Unit FOR 1136, which is gratefully acknowledged. We thank our colleagues in this research unit for several fruitful discussions about our work. 


\section{Notation}

\section{Symbols and Operators}

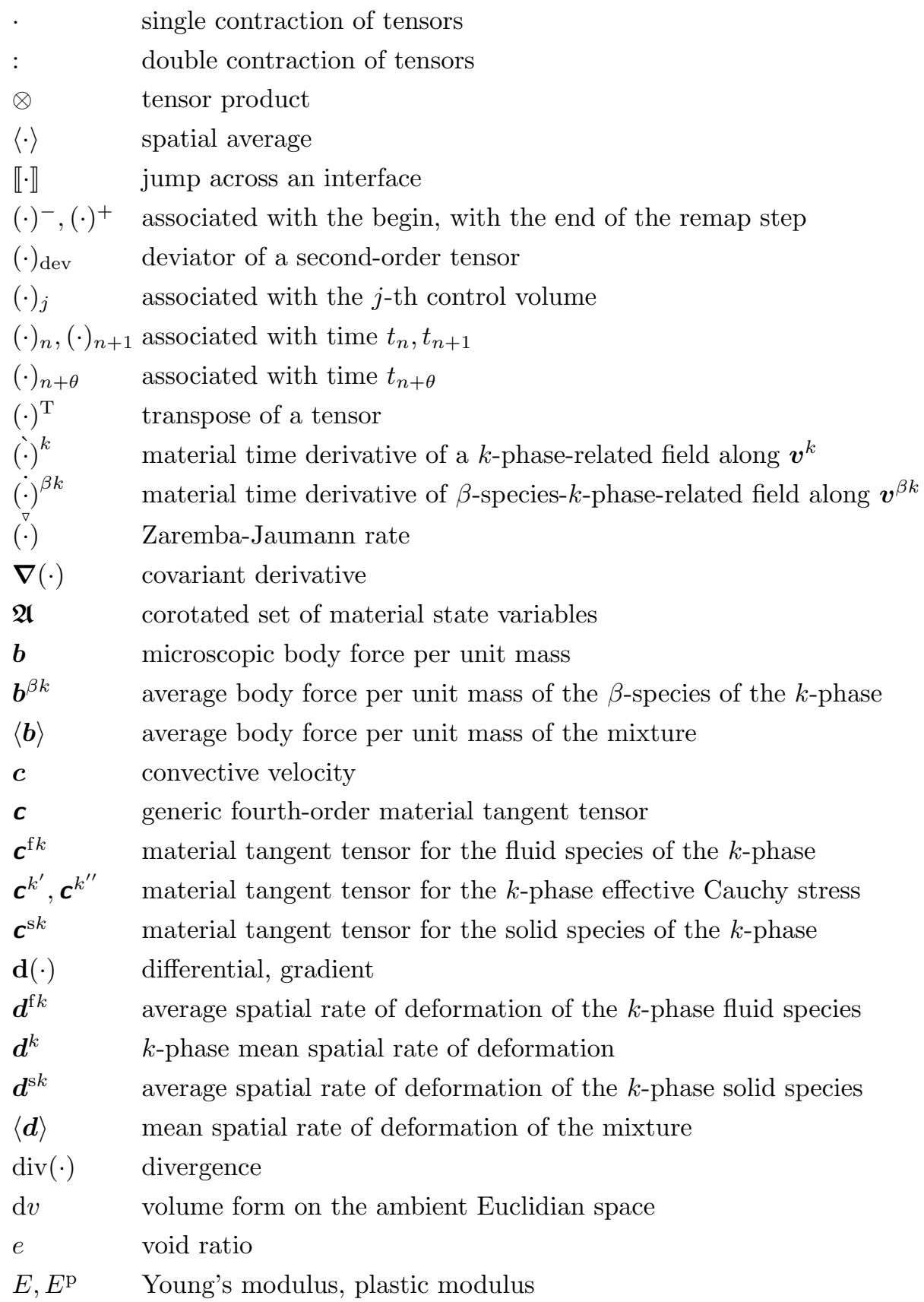


$f^{k} \quad k$-phase volume fraction

$\boldsymbol{f} \quad$ relative incremental deformation gradient

$\mathfrak{f} \quad$ constitutive response function

$\mathrm{Fl}(\cdot) \quad$ averaged convective volume flux

$\boldsymbol{h} \quad$ generic set of material state variables

$\boldsymbol{h}^{k^{\prime}}, \boldsymbol{h}^{k^{\prime \prime}} \quad$ set of material state variables for $k$-phase effective Cauchy stress

$\boldsymbol{h}^{\mathrm{s} k} \quad$ set of material state variables for the solid species of the $k$-phase

I second-order unit tensor

$k \quad$ index for the $k$-th phase of the mixture, $k \in\left\{1, \ldots, n_{\text {mat }}\right\}$

$K \quad$ microscopic bulk modulus

$K^{\mathrm{f} k} \quad$ average bulk modulus of the fluid species of the $k$-phase

$K^{k} \quad$ average $k$-phase bulk modulus (undrained)

$K_{\mathrm{d}}^{k} \quad$ average $k$-phase bulk modulus (drained)

$K_{\mathrm{uj}}^{k} \quad$ average $k$-phase unjacketed bulk modulus

$K^{\text {sk }} \quad$ average bulk modulus of the solid species of the $k$-phase

$\langle K\rangle \quad$ mean bulk modulus of the mixture

$l_{\text {macro }} \quad$ characteristic length at the macroscale

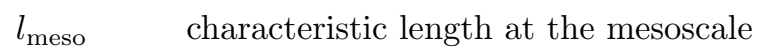

$l_{\text {micro }} \quad$ characteristic length at the microscale

$n^{k} \quad k$-phase fluid fraction, $k$-phase porosity

$n_{\text {mat }} \quad$ number of material phases in the mixture

$\boldsymbol{n}^{*} \quad$ outward normals on an interface

$p \quad$ microscopic pressure

$p^{\mathrm{f} k} \quad$ average pressure in the fluid species of the $k$-phase

$p^{k} \quad$ average $k$-phase pressure

$p^{k^{\prime}}, p^{k^{\prime \prime}} \quad k$-phase mean effective stress

$p^{\text {sk }} \quad$ average pressure in the solid species of the $k$-phase

$p^{\beta k} \quad$ average pressure in the $\beta$-species of the $k$-phase

$P^{k} \quad$ phase function, indicator function

$P^{k} S^{\beta} \quad$ phase-species function, indicator function

$q \quad$ generic scalar-, vector-, or tensor-valued microscopic spatial field

$\hat{q} \quad$ referential or ALE description of the field $q$

$\mathfrak{R} \quad$ rotation related to a corotational rate

$s \quad$ microscopic extra stress

$s^{\mathrm{f} k} \quad$ average extra stress in the fluid species of the $k$-phase

$s^{k} \quad$ average $k$-phase extra stress 
$\boldsymbol{s}^{k^{\prime}}, \boldsymbol{s}^{k^{\prime \prime}} \quad k$-phase effective extra stress

$s^{\text {sk }} \quad$ average extra stress in the solid species of the $k$-phase

$s^{\beta k} \quad$ average extra stress in the $\beta$-species of the $k$-phase

$\langle s\rangle \quad$ average extra stress of the mixture

$S^{\beta} \quad$ species function, indicator function

$\mathcal{S} \quad$ ambient Euclidian space

$\mathfrak{S} \quad$ corotated Cauchy stress

$t \quad$ time

$t_{n}, t_{n+1}$ time at the beginning, at the end of a time step

$t_{n+\theta} \quad$ intermediate time in a time step, with $\theta \in[0,1]$

$\operatorname{tr}(\cdot) \quad$ trace of a second-order tensor

$\boldsymbol{u} \quad$ mean spatial displacement field of the mixture

$\boldsymbol{v} \quad$ microscopic spatial velocity

$\boldsymbol{v}^{\mathrm{f} k} \quad$ mean spatial velocity of the fluid species of the $k$-phase

$\boldsymbol{v}_{\mathrm{I}} \quad$ interface velocity

$\boldsymbol{v}^{k} \quad k$-phase mean spatial velocity

$\boldsymbol{v}^{\text {sk }} \quad$ mean spatial velocity of the solid species of the $k$-phase

$\boldsymbol{v}^{\beta k} \quad$ mean spatial velocity of the $\beta$-species of the $k$-phase

$\langle\boldsymbol{v}\rangle \quad$ mean spatial velocity of the mixture

$V^{\prime} \quad$ volume measure of $\mathcal{V}^{\prime}$ in the ambient Euclidian space

$V_{j} \quad$ volume measure of the $j$-th control volume

$\mathcal{V} \quad$ spatial domain of interest

$\mathcal{V}^{\prime} \quad$ representative volume element (RVE)

$x, x^{\prime} \quad$ points in the ambient Euclidian space

$z \quad$ penetration depth

$\alpha^{k} \quad k$-phase Biot-Willis coefficient

$\beta \quad$ index for the $\beta$-th species of the mixture, $\beta \in\{\mathrm{s}, \mathrm{f}\}$

$\Gamma^{\beta k} \quad$ momentum transfer onto the $k$-phase $\beta$-species due to drag forces

$\delta_{\mathrm{I}}^{\beta k} \quad$ Dirac delta picking out the $\beta$-species- $k$-phase interface

$\Delta \mathfrak{E} \quad$ corotated algorithmic finite strain increment

$\Delta \boldsymbol{r} \quad$ algorithmic finite rotation increment

$\Delta \mathfrak{R} \quad$ incremental rotation

$\Delta \mathfrak{S} \quad$ corotated Cauchy stress increment

$\Delta t \quad$ time increment

$\Delta \varepsilon \quad$ algorithmic finite strain increment

$\dot{\varepsilon}_{\mathrm{vol}}^{\mathrm{s}} \quad$ average volumetric strain rate of solid grains 


$\begin{array}{ll}\Pi^{\beta k} & \text { mass transfer onto the } \beta \text {-species of the } k \text {-phase } \\ \rho & \text { microscopic spatial mass density } \\ \rho^{\mathrm{f} k} & \text { average mass density of the fluid species of the } k \text {-phase } \\ \rho^{k} & \text { average } k \text {-phase mass density } \\ \rho^{\mathrm{s} k} & \text { average mass density of the solid species of the } k \text {-phase } \\ \rho^{\beta k} & \text { average mass density of the } \beta \text {-species of the } k \text {-phase } \\ \langle\rho\rangle & \text { average spatial mass density of the mixture } \\ \sigma^{\mathrm{y}}, \sigma^{\mathrm{y} 0} & \text { yield stress, initial yield stress in uniaxial tension } \\ \boldsymbol{\sigma} & \text { microscopic Cauchy stress } \\ \boldsymbol{\sigma}^{\mathrm{f} k} & \text { average Cauchy stress in the fluid species of the } k \text {-phase } \\ \boldsymbol{\sigma}^{k} & \text { average } k \text {-phase Cauchy stress } \\ \boldsymbol{\sigma}^{k^{\prime}}, \boldsymbol{\sigma}^{k^{\prime \prime}} & k \text {-phase effective Cauchy stress } \\ \boldsymbol{\sigma}^{\mathrm{s} k} & \text { average Cauchy stress in the solid species of the } k \text {-phase } \\ \boldsymbol{\sigma}^{\beta k} & \text { average Cauchy stress in the } \beta \text {-species of the } k \text {-phase } \\ \langle\boldsymbol{\sigma}\rangle & \text { average Cauchy stress of the mixture } \\ \Phi & \text { relative motion of the ALE reference domain } \\ \boldsymbol{\omega} & \text { vorticity tensor }\end{array}$

\section{Abbreviations}

ALE Arbitrary Lagrangian-Eulerian

CFD Computational Fluid Dynamics

FEM Finite Element Method

MMALE Multi-Material Arbitrary Lagrangian-Eulerian

PIV Particle Image Velocimetry

RI-pile vibro-injection pile ("Rüttelinjektionspfahl")

RVE Representative Volume Element

\section{References}

1. D. Aubram. Differential Geometry Applied to Continuum Mechanics. Number 44 in Veröffentlichungen des Grundbauinstitutes der Technischen Universität Berlin. Shaker Verlag, Aachen, 2009. 
2. D. Aubram. An Arbitrary Lagrangian-Eulerian Method for Penetration into Sand at Finite Deformation. Number 62 in Veröffentlichungen des Grundbauinstitutes der Technischen Universität Berlin. Shaker Verlag, Aachen, 2013.

3. D. Aubram. Three-Scale Mechanical Theory for Multi-Material Flow using Spatial Averaging (in preparation).

4. D. Aubram, F. Rackwitz, and S. A. Savidis. An ALE Finite Element Method for Cohesionless Soil at Large Strains: Computational Aspects and Applications. In T. Benz and S. Nordal (eds.): Proceedings 7th European Conference on Numerical Methods in Geotechnical Engineering (NUMGE), pages 245-250. CRC Press, Boca Raton, 2010.

5. T. Belytschko, W. K. Liu, and D. Moran. Nonlinear Finite Elements for Continua and Structures. John Wiley \& Sons, Ltd., 2000.

6. L. S. Bennethum and J. H. Cushman. Multiscale, Hybrid Mixture Theory for Swelling Systems. 1. Balance Laws. International Journal of Engineering Science, 34:125-145, 1996.

7. L. S. Bennethum and T. Weinstein. Three Pressures in Porous Media. Transport in Porous Media, 54:1-34, 2004.

8. L. S. Bennethum. Compressibility Moduli for Porous Materials Incorporating Volume Fraction. Journal of Engineering Mechanics, 132:1205-1214, 2006.

9. L. S. Bennethum. Theory of Flow and Deformation of Swelling Porous Materials at the Macroscale. Computers and Geotechnics, 34:267-278, 2007.

10. D. J. Benson. Computational Methods in Lagrangian and Eulerian Hydrocodes. Computer Methods in Applied Mechanics and Engineering, 99:235-394, 1992.

11. D. J. Benson. A Mixture Theory for Contact in Multi-Material Eulerian Formulations. Computer Methods in Applied Mechanics and Engineering, 140:59-86, 1997.

12. M. A. Biot. General Theory of Three-Dimensional Consolidation. Journal of Applied Physics, 12:155-164, 1941.

13. M. A. Biot and D. G. Willis. The Elastic Coefficients of the Theory of Consolidation. Journal of Applied Mechanics, 24:594-601, 1957.

14. J. A. Bouré and J. M. Delhaye. General Equations and Two-Phase Flow Modeling Section 1.2 in G. Hetsroni (ed.): Handbook of Multiphase Systems. Hemisphere Publishing Corporation, 1982.

15. J. A. Bouré. Two-Phase Flow Models: The Closure Issue. Multiphase Science and Technology, 3(1-4):3-30, 1987.

16. R. M. Bowen. Compressible Porous Media Models by Use of the Theory of Mixtures. International Journal of Engineering Science, 20(6):697-735, 1982.

17. P. Colella, H. M. Glaz, and R. E. Ferguson. Multifluid Algorithms for Eulerian Finite Difference Methods. 1997 (unpublished manuscript).

18. R. De Boer. Theory of Porous Media. Springer-Verlag, Berlin, 2000.

19. E. Detournay and A. H.-D. Cheng. Fundamentals of Poroelasticity Chapter 5 in Comprehensive Rock Engineering: Principles, Practice and Projects. Volume 2: Analysis and Design Methods. Pergamon Press, 1993.

20. D. A. Drew. Mathematical Modeling of Two-Phase Flow. Annual Review of Fluid Mechanics, 15:261-291, 1983.

21. D. S. Drumheller. A Theory for Dynamic Compaction of Wet Porous Solids. International Journal of Solids and Structures, 23:211-237, 1987.

22. W. Ehlers. Grundlegende Konzepte in der Theorie Porser Medien. Technische Mechanik, 16:63-76, 1996.

23. C. W. Hirt, A. A. Amsden, and J. L. Cook. An Arbitrary Lagrangian-Eulerian Computing Method for all Flow Speeds. Journal of Computational Physics, 14:227253, 1974. 
24. A. K. Kapila, R. Menikoff, J. B. Bdzil, S. F. Son, and D. S. Stewart. Two-Phase Modeling of Deflagration-to-Detonation Transition in Granular Materials: Reduced Equations. Physics of Fluids, 13:3002-3024, 2001.

25. H. L. Koning. Some Observations on the Modulus of Compressibility of Water Proceedings European Conference on Soil Mechanics and Foundation Engineering Problems of Settlement and Compressibility of Soils, Section 1, Wiesbaden, Germany, pages 33-36. Deutsche Gesellschaft fr Erd- und Grundbau e.V., 1963.

26. R. W. Lewis and B. A. Schrefler. The Finite Element Method in the Static and Dynamic Deformation and Consolidation of Porous Media. John Wiley \& Sons, Chichester, 2nd ed., 1998.

27. L. E. Malvern. Introduction to the Mechanics of a Continuous Medium. Prentice Hall, Inc., New Jersey, 1969.

28. J. E. Marsden and T. J. R. Hughes. Mathematical Foundations of Elasticity. Dover Publications, New York, 1994.

29. G. H. Miller and E. G. Puckett. A High-Order Godunov Method for Multiple Condensed Phases. Journal of Computational Physics, 128:134-164, 1996.

30. D. S. Miller and G. B. Zimmerman. An Algorithm for Time Evolving Volume Fractions in Mixed Zones in Lagrangian Hydrodynamics Calculations. Russian Journal of Physical Chemistry B, 3:117-121, 2009.

31. A. Murrone and H. Guillard. A Five Equation Reduced Model for Compressible Two Phase Flow Problems. Journal of Computational Physics, 202:664-698, 2005.

32. M. A. Nikolinakou, A. J. Whittle, S. A. Savidis, and U. Schran. Prediction and Interpretation of the Performance of a Deep Excavation in Berlin Sand. Journal of Geotechnical and Geoenvironmental Engineering, 137(11):1047-1061, 2011.

33. A. Niemunis and I. Herle. Hypoplastic Model for Cohesionless Soils with Elastic Strain Range. Mechanics of Cohesive-Frictional Materials, 2:279-299, 1997.

34. F. Rackwitz and S. A. Savidis. Numerische Untersuchungen zum Tragverhalten von Zugpfhlen in Berliner Sand. Bauingenieur, 79(9):375-383, 2004.

35. S. A. Savidis, D. Aubram, and F. Rackwitz. Arbitrary Lagrangian-Eulerian Finite Element Formulation for Geotechnical Construction Processes. Journal of Theoretical and Applied Mechanics, 38(1-2):165-194, 2008.

36. M. Shashkov. Closure Models for Multimaterial Cells in Arbitrary LagrangianEulerian Hydrocodes. International Journal for Numerical Methods in Fluids 56(8):1497-1504, 2008.

37. H. B. Stewart and B. Wendroff. Two-Phase Flow: Models and Methods. Journal of Computational Physics, 56:363-409, 1984.

38. C. Truesdell and R. A. Toupin. Encyclopedia of Physics. Bd. III/1: The Classical Field Theories, pages 226-793. Springer-Verlag Berlin Gttingen Heidelberg, 1960.

39. C. Truesdell and W. Noll. The Non-Linear Field Theories of Mechanics. SpringerVerlag Berlin Heidelberg New York, 3rd ed., 2004.

40. E. Vitali and D. J. Benson. An Extended Finite Element Formulation for Contact in Multi-Material Arbitrary Lagrangian-Eulerian Calculations. International Journal for Numerical Methods in Engineering, 67:1420-1444, 2006.

41. P.-A. von Wolffersdorff. A Hypoplastic Relation for Granular Materials with a Predefined Limit State Surface. Mechanics of Cohesive-Frictional Materials, 1:251271, 1996.

42. S. Whitaker. The Transport Equations for Multi-Phase Systems. Chemical Engineering Science, 28:139-147, 1973.

43. S. Whitaker. Flow in Porous Media III: Deforming Media. Transport in Porous Media, 1:127-154, 1986 . 
44. P. Wriggers. Nonlinear Finite Element Methods. Springer-Verlag Berlin Heidelberg, 2008.

45. O. C. Zienkiewicz and T. Shiomi. Dynamic Behaviour of Saturated Porous Media: The Generalized Biot Formulation and its Numerical Solution. International Journal for Numerical and Analytical Methods in Geomechanics, 8:71-96, 1984.

46. O. C. Zienkiewicz, A. H. C. Chan, M. Pastor, B. A. Schrefler, and T. Shiomi. Computational Geomechanics - With Special Reference to Earthquake Engineering. John Wiley \& Sons, Chichester, 1999.

47. O. C. Zienkiewicz and R. L. Taylor. The Finite Element Method, 3 vols. Butterworth-Heinemann, 5th ed., 2000. 